\title{
A Planar 3-Convex Set is Indeed a Union of Six Convex Sets
}

\author{
Noa Nitzan · Micha A. Perles
}

Received: 1 July 2010 / Revised: 2 November 2011 / Accepted: 6 January 2012 /

Published online: 20 March 2013

(C) Springer Science+Business Media New York 2013

\begin{abstract}
Suppose $S$ is a planar set. Two points $a, b$ in $S$ see each other via $S$ if $[a, b]$ is included in $S$. F. Valentine proved in 1957 that if $S$ is closed, and if for every three points of $S$, at least two see each other via $S$, then $S$ is a union of three convex sets. The pentagonal star shows that the number three is the best possible. We drop the condition that $S$ is closed and show that $S$ is a union of (at most) six convex sets. The number six is best possible.
\end{abstract}

Keywords Non-convexity · Visually independent · Seeing subset · Invisibility graph · Valentine's Theorem (57')

\section{Introduction}

There are three common measures for evaluating the "non-convexity" of a set $X \subset \mathbb{R}^{d}$ : $\alpha(X)$ - The largest size of a visually independent subset of $X$. $\beta(X)$ - The smallest size of a collection of seeing subsets of $X$ that covers $X$, or, in other words, the chromatic number of the invisibility graph of $X$. $\gamma(X)$ - The smallest size $k$ such that $X$ is a union of $k$ convex sets.

The content of this paper forms part of a Ph.D. thesis written by the first author under the supervision of the second author.

\section{N. Nitzan $(\varangle)$}

Department of Mathematics, Center for the Study of Rationality, The Hebrew University of Jerusalem, Jerusalem, Israel e-mail: noanitzan@ma.huji.ac.il

\section{M.A. Perles}

Department of Mathematics, The Hebrew University of Jerusalem, Jerusalem, Israel e-mail: perles@math.huji.ac.il 
Much effort has been devoted to bounding $\gamma$ in terms of $\alpha$. In general, there is no such bound, since there exist planar sets $X$ with $\alpha(X)=3$ but with $\gamma(X)=\infty$, and there exist closed sets $S \subset \mathbb{R}^{4}$ with $\alpha(S)=2$ and $\gamma(S)=\infty$ (even $\beta(S)=\infty$ ). In the specific case of closed sets in the plane, the situation is different.

Valentine [6] proved that for closed $S \subset \mathbb{R}^{2}, \alpha(S)=2$ implies $\gamma(S) \leq 3$. Eggleston [7] proved that for compact $S \subset \mathbb{R}^{2}, \alpha(S)<\infty$ implies $\gamma(S)<\infty$. Breen and Kay [2] were the first to find an upper bound for $\gamma$ in terms of $\alpha$. They proved that for closed $S \subset \mathbb{R}^{2}$, if $\alpha(S)=m$ then $\gamma(S) \leq m^{3} \cdot 2^{m}$. Later on, Perles and Shela [5] improved this upper bound to $m^{6}$, and Matousek and Valtr [4] obtained the best upper bound know today, $18 \mathrm{~m}^{3}$. In the same paper, they give examples of closed planar sets with $\gamma(S) \mathrm{cm}^{2}$.

There has also been some success in bounding $\gamma$ in terms of $\alpha$ for certain cases of planar sets $X$ that are not necessarily closed. Breen [1] claims that for $X \subset \mathbb{R}^{2}, \alpha(X)=$ 2 implies $\gamma(X) \leq 6$. Another result is of Matousek and Valtr [4] who proved that for $X \subset \mathbb{R}^{2}$ with $\alpha(X)$ finite, if $X$ is starshaped then $\gamma(X) \leq 2(\alpha(X))^{2}$. They also proved that for $X \subset \mathbb{R}^{2}$, if $\mathbb{R}^{2} \backslash X$ has no isolated points then $\gamma(X) \leq(\alpha(X))^{4}$.

In this work we shall focus on the case of $X \subset \mathbb{R}^{2}$ with $\alpha(X)=2$. We wish to complete the work of Breen [1], and give a detailed proof of the theorem claimed by Breen $(\alpha=2 \Rightarrow \gamma \leq 6)$. We intend to determine the maximum possible value of $\gamma(X)$ (assuming $X \subset \mathbb{R}^{2}$ and $\alpha(X)=2$ ) under a variety of side conditions, pertaining to the location (within $\operatorname{cl} X$ ) of the points of $\operatorname{cl} X \backslash X$. We produce examples for all cases under discussion, showing that the bounds obtained are tight.

\section{Definitions and Notations}

Given $X \subseteq \mathbb{R}^{2}$, we say that two points $u, v \in \mathbb{R}^{2}$ see each other via $X$ if the open interval $(u, v)$ is included in $X$. (This applies even if the points $u, v$ are not in $X$.)

$A$ is a seeing subset of $X$ if $A \subseteq X$ and every two points of $A$ see each other via $X$. A subset of $X$ is visually independent if no two of its points see each other via $X$.

Define the invisibility graph of $X$ as the graph $G(X)$ with vertex set $X$ and with $u, v \in X$ connected by an edge iff $[u, v] \nsubseteq X$.

We now define the three most common "measures of non-convexity" of $X$ : (These are the notations found in the literature which we prefer.)

$\alpha(X)$ - The supremum of cardinalities of all visually independent subsets of $X$. That is, the clique number of the graph $G(X)$.

$\beta(X)$ - The chromatic number of $G(X)$. In other words, the smallest cardinality of a collection of seeing subsets of $X$ that covers $X$.

$\gamma(X)$-The smallest cardinality $k$ such that $X$ can be expressed as the union of $k$ convex sets.

It is easy to see that $\alpha(X) \leq \beta(X) \leq \gamma(X)$.

The following notations will be used throughout this paper: For $X \subset \mathbb{R}^{2}$, define $S=\operatorname{cl} X$. We shall write $M=S \backslash X$ ( $M$ is the set of points of $S$ missing in $X)$. We split $M$ into two parts $M=M_{\mathrm{b}} \cup M_{i}$, where $M_{i}=M \cap$ int $S$ and $M_{\mathrm{b}}=M \cap$ bd $S$. 
Table 1 Summary of all results

\begin{tabular}{lll}
\hline & $M_{\mathrm{b}}=\phi$ or $M_{\mathrm{b}}=b d s$ & $M_{b}$ unrestricted \\
\hline$\left|M_{i}\right|>1$ & $2(2)$ & $3(3)$ \\
$\left|M_{i}\right|=0$ & $3(1)$ & $3(3)$ \\
$\left|M_{i}\right|=1$ & $4(2)$ & $6(4)$ \\
\hline
\end{tabular}

$S$ is locally convex at a point $x$ if $x \in S$ and $x$ has a neighborhood $U$ such that $S \cap U$ is convex. We denote by $Q(=\operatorname{lnc} S)$ the set of points of local non-convexity (lnc points) of $S$. These are the points where $S$ fails to be locally convex.

We say that $S$ is 2-dimensional at a point $p$ if $p \in \operatorname{cl}($ int $S$ ).

$A \subset \mathbb{R}^{d}$ is an $L_{2}$-set if every two points of $A$ can be connected by a polygonal line of at most 2 edges within $A$.

Given a subset $S_{0} \subseteq S$, we say that $S_{0}$ is convex relative to $S$ if for every $x, y \in$ $S_{0},[x, y] \subseteq S$ implies $[x, y] \subseteq S_{0}$.

\section{Results}

Throughout the following theorems we assume that $X$ is a planar set, $\alpha(X) \leq 2$, and that $S=\operatorname{cl} X$.

Main Theorem $1 \max \left\{\gamma(X): X \subseteq \mathbb{R}^{2}, \alpha(X) \leq 2\right\}=6$.

We disassemble Main Theorem 1 into several independent theorems: Theorems $\mathrm{A}-\mathrm{G}$ :

Theorem A If $X$ is not an $L_{2}$-set (in particular, if $X$ is not connected), then $\gamma(X)=2$. (In this theorem, $\mathbb{R}^{2}$ can be replaced by an arbitrary real vector space.)

Theorem B If $S$ is not 2-dimensional at some point $p \in S$, then $\gamma(X) \leq 2$.

Theorem C If $\left|M_{i}\right|>1$, then $\gamma(X) \leq 3$. The number three is the best possible, even when $S$ is convex. If, in addition, $M_{\mathrm{b}}=\phi$ or $M_{\mathrm{b}}=\mathrm{bd} S$, then $\gamma(X)=2$.

Theorem D If $\left|M_{i}\right|=1$ and $M_{\mathrm{b}}=\phi$ or $M_{\mathrm{b}}=$ bd $S$, then $\gamma(X) \leq 4$. The number four is the best possible.

Theorem $\mathbf{E}$ If $M_{i}=\phi$ then $\gamma(X) \leq 3$. The number three is the best possible, even when $S$ is convex.

Theorem $\mathbf{F}$ If $\left|M_{i}\right|=1$ then $\gamma(X) \leq 6$. The number six is the best possible.

Theorem $\mathbf{G}$ If $\left|M_{i}\right|=1$ and $S$ is convex then $\gamma(X) \leq 4$. The number four is the best possible. If, in addition, $M_{\mathrm{b}}=\phi$ or $M_{\mathrm{b}}=$ bd $S$ then $\gamma(X)=2$.

Table 1 summarizes all the cases above. In each box appears $\max \gamma(X)$ under the conditions of that box. The number in parentheses is $\max \gamma(X)$ under the conditions of the box together with the extra assumption that $S$ is convex.

Much of the material contained in this paper can be summarized in the following extension of Valentine's Theorem: 
Theorem 3.1 If $X \subseteq \mathbb{R}^{2}, \alpha(X) \leq 2$, and the complement $\mathbb{R}^{2} \backslash X$ has no one-pointed components, then $\gamma(X) \leq 3$.

We also present two results and a conjecture involving the measure $\beta$ :

Main Theorem $2 \max \left\{\gamma(X): X \subseteq \mathbb{R}^{2}, \beta(X)=2\right\}=4$.

It seems that Main Theorem 2 has been known for many years.

Example 8 We present a bounded set $X \subset \mathbb{R}^{2}$ with $\alpha(X)=2$ and $\beta(X)=4$.

Conjecture $\max \left\{\beta(X): X \subseteq \mathbb{R}^{2}, \alpha(X)=2\right\}=4$.

\section{Proof of Theorem A}

As $X$ is not an $L_{2}$-set, there are two points $a, b \in X$ that cannot be connected by a polygonal line of fewer than 3 edges within $X$. In other words, there is no point in $X$ that sees both $a$ and $b$. Define $A=s t(a)=\{x \in X:[a, x] \subseteq X\}, B=s t(b)$. Notice that the sets $A$ and $B$ are disjoint. We show now that $A$ is convex: Take $p, q \in A$. If $p^{\prime} \in(a, p], q^{\prime} \in(a, q]$, then $\left[p^{\prime}, q^{\prime}\right] \subset X$, since otherwise $\left\{p^{\prime}, q^{\prime}, b\right\}$ would be a visually independent set. Hence the full triangle $[a, p, q]$ is included in $X$, so $a$ sees via $X$ every point in $[p, q]$, which means that $[p, q] \subset A$, so $A$ is convex. Similarly, $B$ is convex. Now, for every $x \in X, x \in A \cup B$, because otherwise $\{a, b, x\}$ would be a visually independent set. Thus $X$ is the union of two disjoint convex sets.

\section{Proof of Theorem B}

$S$ is a closed set in the plane and, therefore, according to Valentine [6], is a union of at most three convex sets: $S=\cup_{i=1}^{n} C_{i}$, where $1 \leq n \leq 3$. As $S$ is closed, we can assume that for each $i, C_{i}$ is closed. In addition, we will assume that none of these convex sets are included in the union of the others.

If each $C_{i}$ is 2-dimensional then $S$ is 2-dimensional. Assume, therefore, w.l.o.g., that $C_{1}$ is not 2-dimensional. If $\operatorname{dim} C_{1}=0$, then $S$ is not connected and, therefore, $X$ is not connected, so by Theorem A, $\gamma(X) \leq 2$. Otherwise, $\operatorname{dim} C_{1}=1$, in which case $C_{1}$ is part of a line $L$. There is a point $p \in C_{1}$ such that $p \notin C_{2} \cup C_{3}$. Since $C_{2}, C_{3}$ are closed, there is a neighborhood $U$ of $p$ that misses $C_{2} \cup C_{3}$, and, therefore, $U \cap S=U \cap C_{1}$ is a segment. Define $L$ to be the line containing this segment. Denote by $L_{+}, L_{-}$the open half-planes determined by $L$.

Define $C=\operatorname{conv}(X \backslash L)$. We wish to show that $C \subseteq X$. Every two points in $X \cap L_{+}$do not see $p$ via $X$. Therefore, since $\alpha(X)=2$, they see each other via $X \cap L_{+}$. Hence, $X \cap L_{+}$is convex. By the same argument, $X \cap L_{-}$is convex, so $C=\operatorname{conv}(X \backslash L)=\operatorname{conv}\left(\left(X \cap L_{+}\right) \cup\left(X \cap L_{-}\right)\right)$.

If $X \cap L_{+}=\phi$ (or $X \cap L_{-}=\phi$ ) then $C=X \cap L_{-} \subseteq X$ (or $C=$ $X \cap L_{+} \subseteq X$ ). If both $X \cap L_{+}$and $X \cap L_{-}$are nonempty, then $C=\cup\{[a, b]$ : $\left.a \in X \cap L_{+}, b \in X \cap L_{-}\right\}$. The point $p$ does not see any $a \in L_{+} \cap X$ or $b \in L_{-} \cap X$. Therefore, again, as $\alpha(X)=2$, for any such $a, b,[a, b] \subset X$. This implies that $C \subset X$. 
It remains to deal with the set $L \cap X$. Since $\alpha(X)=2$ and $L$ is convex, $\alpha(X \cap L) \leq 2$. If $X \cap L$ is convex, we are done. Otherwise, $X \cap L$ is the disjoint union of two nonempty convex sets $A, B$, where, say, $p \in A$. If $C=\phi$ then we are done, so assume $C \neq \phi$.

In order to complete the proof, we would like to show that $\operatorname{conv}(B \cup C) \subset X$. Since both $B$ and $C$ are convex, $\operatorname{conv}(B \cup C)=\cup\{[b, c]: b \in B, c \in C\}$. Suppose $b \in B$ and $c \in C$ :

Case 1 If $c \notin L$ then $[p, c] \nsubseteq X$ and $[p, b] \nsubseteq X$, hence $[b, c] \subset X$.

Case 2 If $c \in L$ then $c \in\left[c_{+}, c_{-}\right]$, where $c_{+} \in X \cap L_{+}$and $c_{-} \in X \cap L_{-}$. The points $c_{+}, c_{-}, b$ do not see $p$ via $X$, therefore, and since $\alpha(X)=2,\left[c_{+}, b\right] \subset X$ and $\left[c_{-}, b\right] \subset X$. Now, each point in $[c, b)$ is in the convex hull of a point in $\left[c_{+}, b\right)$ and a point in $\left[c_{-}, b\right)$ and, therefore, is in $X$ (again, these two points do not see $p$, and $\alpha(X)=2)$.

This establishes that $\operatorname{conv}(B \cup C) \subset X$, which implies that $X$ is the union of two convex sets: $A$, the component of $p$ in $L \cap X$, and $\operatorname{conv}(B \cup C)(=B \cup C)$.

\section{Proof of Theorem C}

Coming to prove theorem $\mathrm{C}$, we shall first show that if $\left|M_{i}\right|>1$, then $M_{i}$ contains a segment. Suppose $x, y \in M_{i}, x \neq y$, and let $L$ be the line spanned by $x, y$. As $x, y \in$ int $S$, both have circular neighborhoods $U_{x}, U_{y}$ in $S$. The intersection of $L \backslash\{x, y\}$ with these two neighborhoods consists of 4 segments. These segments lie in the three components of $L \backslash\{x, y\}$, and, therefore, at least one of them is disjoint from $X$. Therefore, $M_{i}$ includes a segment, call it $I$, and $L=\operatorname{aff} I$.

Denote by $L_{+}, L_{-}$the open half-planes determined by $L$. Define $X_{+}=X \cap$ $L_{+}, X_{-}=X \cap L_{-}$and $S_{+}=\operatorname{cl}\left(X_{+}\right), S_{-}=\operatorname{cl}\left(X_{-}\right)$. Next we show that $X_{+}$is convex. Take $p, q \in X_{+}$. There is a point $y \in X_{-}$, close enough to the center of $I$, such that both segments $(p, y),(q, y)$ intersect $I$, meaning that $y$ sees neither $p$ nor $q$ via $X$, and, therefore, $[p, q] \subset X$, hence $[p, q] \subset X_{+}$. Similarly, $X_{-}$is convex.

Next we show that $M_{i} \subset L$. Note that $S \cap L_{+} \subset \operatorname{cl} X_{+}$, and, therefore, $L_{+} \cap$ int $S=\operatorname{int}\left(S \cap L_{+}\right) \subset$ int $\operatorname{cl} X_{+}=\operatorname{int} X_{+} \subset X$. Therefore, $L_{+} \cap M_{i}=\phi$. Similarly, $L_{-} \cap M_{i}=\phi$, hence $M_{i} \subset L$.

Define: $B_{+}=S_{+} \cap L, B_{-}=S_{-} \cap L . B_{+}$is an edge of $S_{+}$(think of it as the base of $S_{+}$). If a point $u$ lies in rel int $B_{+}$, then $u$ sees every point of $S \cap L_{+}$via int $S_{+}$, hence via $X$. Thus, a point $u \in X \cap B_{+}$may fail to see some point of $X_{+}$via $X$ only if $u$ is an endpoint of $B_{+}$. Similarly for $B_{-}$and $X_{-}$.

If $X$ contains a point $y$ that is in $L \backslash\left(B_{-} \cup B_{+}\right)$, then $S$ is not 2-dimensional at y, and, therefore, $\gamma(X)=2$, by Theorem B. Assume, therefore, that $X \cap L \subset B_{-} \cup B_{+}$. Note that the segment $I\left(\subset M_{i}\right)$ lies in $B_{-} \cap B_{+}$.

Next we show that $\gamma(X)=2$, unless $X \cap L \subset B_{-} \cap B_{+}$. Assume $X \cap L \nsubseteq B_{-} \cap B_{+}$. Pick a point $y \in X \cap L \backslash\left(B_{-} \cap B_{+}\right)$. Think of $L$ as a horizontal line, and suppose, w.l.o.g., that $y \notin B_{-}$, and that $y$ is to the right of $B_{-}$.

Denote by $L_{2}$ the component of $y$ in $X \cap L . L_{1}$ is the other component of $X \cap L$, if $X \cap L$ is not convex. If $X \cap L$ is convex, then $L_{1}=\phi$.

Clearly, $y$ does not see any point of $L_{1}$ via $X$. Since $y \notin B_{-}, y$ does not see any point of $X_{-}$via $X$. Since $\alpha(X)=2$, every point of $L_{1}$ sees every point of $X_{-}$via $X$, 
hence via $X_{-}$. In other words, $L_{1} \cup X_{-}$is convex. But, as we shall see immediately, $L_{2} \cup X_{+}$is convex as well. Indeed, consider a point $x \in X_{+}$and a point $y^{\prime} \in L_{2}$, to the right of $y\left(y^{\prime}=y\right.$ included). $x$ does not see via $X$ some point $z \in X_{-}$, that lies beyond $I . y^{\prime} \notin B_{-}$and, therefore, does not see via $X$ any point in $X_{-}$. It follows that $y^{\prime}$ sees $x$ via $X$. Now consider a point $y^{\prime \prime} \in L_{2}$, strictly to the left of $y$. Since $y^{\prime \prime} \in L_{2}$ lies to the right of $I$, and $I \subset B_{+}$, we conclude that $y^{\prime \prime} \in \operatorname{rel} \operatorname{int} B_{+}$, and, therefore, sees via $X_{+}$every point of $X_{+}$. Now we can represent $X$ as the union of two convex sets: $X=\left(L_{1} \cup X_{-}\right) \cup\left(L_{2} \cup X_{+}\right)$.

Assume from now on that $X \cap L \subset B_{-} \cap B_{+}$. Let us first dispose of the case where $M_{\mathrm{b}}=\phi$ or $M_{\mathrm{b}}=$ bd $S$.

$M_{\mathrm{b}}=\phi$ : If $c \in X \cap L\left(\subset B_{-} \cap B_{+}\right)$then $c$ sees every point of $X_{+}$via $S \cap L_{+}$, which is a subset of $X_{+}$. Same for $X_{-}$and $L_{-}$. Denote by $L_{1}, L_{2}$ the components of $X \cap L$. ( $L_{1}=\phi$ if $X \cap L$ is convex). Then $X$ is the union of the two convex sets $L_{1} \cup X_{+}, L_{2} \cup X_{-}$.

$M_{\mathrm{b}}=$ bd $S$ : If $x \in X \cap L$ then $x \in \operatorname{rel}$ int $B_{+}$. (The endpoints of $B_{+}$are boundary points of $S$ and, therefore, not in $X$.) Similarly, $x \in \operatorname{rel}$ int $B_{-}$. Define $L_{1}, L_{2}$ as above. Then $X$ is again the union of the two convex sets $L_{1} \cup X_{+}$and $L_{2} \cup X_{-}$.

Now we return to the general case: $\left|M_{i}\right|>1$ and $M_{\mathrm{b}}$ unrestricted, and try to show that $\gamma(X) \leq 3$.

If $X \cap L$ is convex then $X$ is the union of three convex sets and we are done. Assume $X \cap L$ is not convex, so it is the union of two non-empty components $L_{1}, L_{2}$, where $L_{1}$ is to the left of $L_{2}$. If $L_{1}$ has no left endpoint, then $L_{1} \subset$ rel int $B_{+}$, and, therefore, $X$ is the union of the three convex sets $L_{1} \cup X_{+}, X_{-}, L_{2}$. The same argument works when $L_{1}$ has a left endpoint $c_{1}$, but $c_{1}$ is not the left endpoint of $B_{+}$. We can repeat this argument with $B_{-}, X_{-}$instead of $B_{+}, X_{+}$, and also with $L_{2}$ instead of $L_{1}$.

Assume, therefore, that $L_{1}$ has a left endpoint $c_{1}, L_{2}$ has a right endpoint $c_{2}$, and $B_{+}=B_{-}=\left[c_{1}, c_{2}\right]$. The point $c_{1}$ still sees every point of $S \cap L_{+}$via int $S_{+}(\subset X)$ unless $S_{+}$has an edge $C_{1_{+}}$with endpoint $c_{1}$, other than $B_{+}$. Assume, therefore, that $S_{+}$has such an edge $C_{1_{+}}$, and, by the same token, that $S_{+}$has an edge $C_{2_{+}}$with endpoint $c_{2}$, other than $B_{+}$(see Fig. 1). If $X \cap C_{1_{+}}$is convex then $c_{1}$ still sees every point of $X_{+}$via $X_{+}$, and thus $L_{1} \cup X_{+}$is again convex, as before.

Assume, therefore, that $X \cap C_{1_{+}}$is not convex. It is the union of $\left\{c_{1}\right\}\left(=C_{1_{+}} \cap L\right)$ and the convex set $C_{1_{+}} \cap X_{+}$. By the same token, assume that $X \cap C_{2_{+}}$is not convex. It follows that $X \cap C_{1_{+}} \cap C_{2_{+}}=\phi$ since a point $z \in X \cap C_{1_{+}} \cap C_{2_{+}}$would form a 3 -clique of invisibility with $c_{1}$ and $c_{2}$.

Fig. 1 Here $\mathrm{X} \cap \mathrm{L}$ is not convex

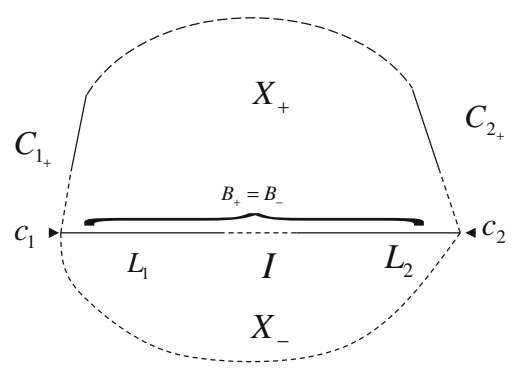



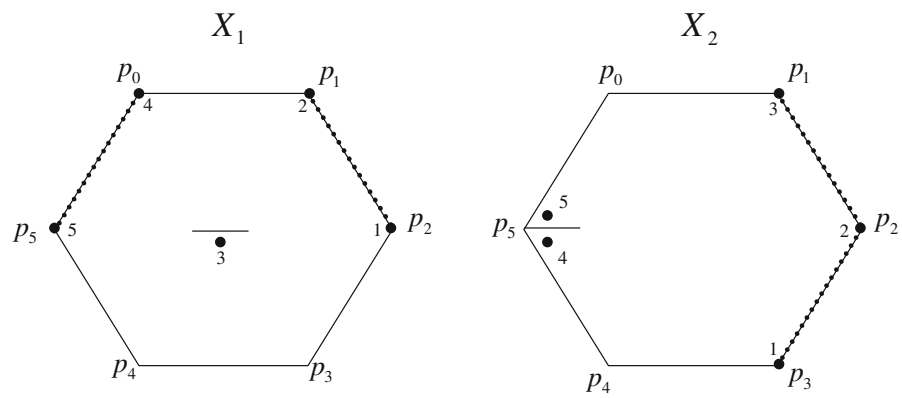

Fig. 2 Examples where $\left|M_{i}\right|>1, \alpha=2$ and $\gamma=3$

We could play the same game with $X_{-}$, but this is not necessary, since $X$ is the union of the three convex sets $X_{-},\left(X_{+} \backslash C_{1_{+}}\right) \cup L_{1},\left(X_{+} \backslash C_{2_{+}}\right) \cup L_{2}$.

Examples 1, 2 show that the number three is the best possible: We describe two sets $X_{1}, X_{2} \subset \mathbb{R}^{2}$ with $\left|M_{i}\right|>1$ and show that $\alpha$ of each set is 2 and that $\gamma$ of each set is three. Notice that $X_{1} \cap L$ is not convex, while $X_{2} \cap L$ is convex.

Example 1 Let $P$ be a regular hexagon with center $O$ and vertices $p_{0}, p_{1}, \ldots, p_{5}$. Take $[a, b]$ to be a short segment lying on $\left[p_{5}, p_{2}\right]$ with $O$ in its center. We define $X_{1}=P \backslash\left(\left(p_{5}, p_{0}\right) \cup\left(p_{1}, p_{2}\right) \cup[a, b]\right)$ (see Fig. 2).

$\alpha\left(X_{1}\right)=2$ : The set $X_{1} \backslash\left\{p_{0}\right\}$ is the union of two convex sets. The same holds for $X_{1} \backslash\left\{p_{1}\right\}$. Therefore, if there is a 3-circuit of invisibility in $X_{1}$, it must contain both $p_{0}$ and $p_{1}$. But these two points see each other via $X_{1}$.

$\gamma\left(X_{1}\right) \geq 3$ since, as shown in Fig. 2, there is a 5-circuit of invisibility.

Example 2 Let $P$ be as above and take $[c, d]$ to be a short segment lying on $\left[p_{5}, p_{2}\right]$ with $c=p_{5}$. Define $X_{2}=P \backslash\left(\left(p_{1}, p_{2}\right) \cup\left(p_{2}, p_{3}\right) \cup[c, d]\right)$ (see Fig. 2).

$\alpha\left(X_{2}\right)=2$ : The set $X_{2} \backslash\left\{p_{2}\right\}$ is the union of two convex sets. Therefore, if there is a 3-clique of invisibility in $X_{2}$, it must contain $p_{2}$. But there are only two points that $p_{2}$ does not see via $X_{2}: p_{1}$ and $p_{3}$, and fortunately $\left[p_{1}, p_{3}\right] \subset X_{2}$.

$\gamma\left(X_{2}\right) \geq 3$ since, as shown in Fig. 2, there is a 5-circuit of invisibility.

\section{Proof of Theorem D}

Since Theorem $D$ is not needed for the proof of the remaining theorems, we skip the proofs, and only give an example to show that the number 4 is the best possible. The interested reader may find a proof (even two proofs) of Theorem D in the detailed version (www.ma.huji.ac.il/ noanitzan/non-convexity.pdf, p. 10, Sect. 7).

A well-known example shows that the number four is the best possible.

Example 3 We describe a set $X \subset \mathbb{R}^{2}$ with $\left|M_{i}\right|=1$ and $M_{\mathrm{b}}=\phi$, and show that $\alpha(X)=2$ and $\gamma(X) \geq 4$. Let $X$ be a closed Star of David with its center $O$ removed. Denote by $p_{0}, p_{1}, \ldots, p_{5}$ the outer vertices of the Star of David (see the left side of Fig. 3). $\alpha(X)=2$ : The right side of Fig. 3 shows a representation of $X$ as the union of two seeing subset, hence $\beta(X)=2$. Therefore, $\alpha(X)=2$. 

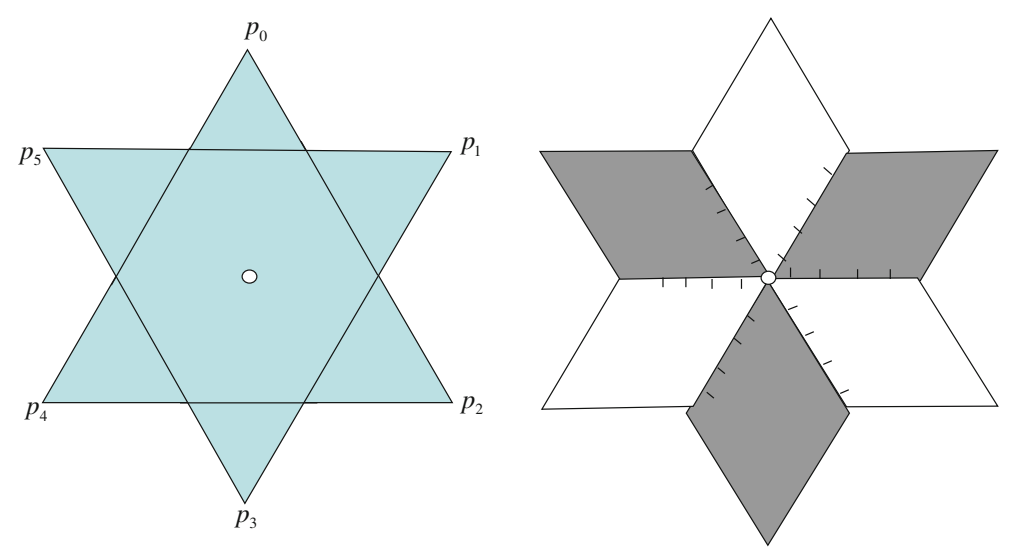

Fig. 3 An example where $\left|M_{i}\right|=1, M_{b}=\phi, \alpha=2$ and $\gamma=4$

$\gamma(X) \geq 4$ : Let $C$ be a convex subset of $X$. Define $A=\left\{p_{0}, p_{2}, p_{4}\right\}$ and $B=$ $\left\{p_{1}, p_{3}, p_{5}\right\}$. No point of $A$ sees any point of $B$, therefore, $C$ cannot contain points of both sets. $A \nsubseteq C$ and $B \nsubseteq C$, since $O \notin C$. Therefore, $C$ contains at most two points of $A$ or two points of $B$. Hence, we need at least two convex subsets of $X$ to cover $A$, and two other convex subsets of $X$ to cover $B$. It follows that $\gamma(X) \geq 4$. One can easily represent $X$ as a disjoint union of 4 convex sets.

For an example with $\left|M_{i}\right|=1$ and $M_{\mathrm{b}}=\mathrm{bd} S$, take the same set $X$ and remove its boundary.

\section{Proof of Theorem E, Preliminary Considerations}

Let us recall our assumptions: $X \subset \mathbb{R}^{2}, \alpha(X)=2, S=\operatorname{cl} X$ (hence $\alpha(S) \leq 2$ and, therefore, $\gamma(S) \leq 3$, by [6]), $M_{i}=\phi$, which means just that int $S \subset X \subset S$. We wish to show that $\gamma(X) \leq 3$. We first need the following lemmata:

Lemma 8.1 S has no triangular holes.

Proof If $S$ is convex, then of course, there are no holes in $S$.

If $S$ is not connected, Then $S$ is the union of two disjoint, closed convex sets, and again there are no holes in $S$.

Otherwise, if $S$ is connected but not convex, then by Tietze's Theorem, $S$ contains an $\operatorname{lnc}$ point $q$. According to [6], $q \in \operatorname{ker} S$. In other words, $S$ is starshaped with respect to $q$. But a starshaped set has no holes.

Lemma 8.2 If $M_{i}=\phi$, then $\beta(X)=\gamma(X)$.

Proof It suffices to show that if $A$ is a seeing subset of $X$ then $\operatorname{conv} A \subset X$. Every point in $\operatorname{conv} A$ is a convex combination of at most three points of $A$. If $x$ is a convex combination of two points of $A$, then $x \in X$. Assume $x$ is a convex combination of three affinely independent points $a, b, c \in A$. The edges of the triangle $\Delta=[a, b, c]$ 
lie in $X$. By Lemma 8.1, $S$ has no triangular holes, therefore, $\Delta \subset S$. This implies $x \in$ int $\Delta \subset$ int $S \subset X$.

In view of Lemma 8.2, we only have to find how many seeing subsets of $X$ are needed in order to cover $X$.

For the rest of the proof, we distinguish four cases, according to the dimension of the kernel $K=\operatorname{ker} S$ : Case 1: $K=\phi$, Case 2: $K$ is a single point, Case 3: $\operatorname{dim} K=1$, Case $4: \operatorname{dim} K=2$. The first three cases are relatively simple, and are treated in full in the detailed version (www.ma.huji.ac.il/ noanitzan/non-convexity.pdf, p. 15, Sect. 8). Here we describe only the more interesting case, Case 4.

\section{Proof of Theorem E, Reduction to the Polygonal Case}

As mentioned above, the case $\operatorname{dim} K=2$ is the most complicated case of the four, which Breen [1] relates to lengthy. She claims that in this case, $X$ is the union of four convex sets. We will show that $X$ is the union of three convex sets. This result can be viewed as the focal point of the whole paper, since it trivially implies theorem $\mathrm{F}$.

Stage 1: Reduction to the polygonal case:

In this section we intend to show why it is possible to assume that $S$ is a compact, polygonal set. Our approach depends heavily on the following important result of [3]:

Let $T$ be a subset of a real vector space. Assume that every finite subset $F \subseteq T$ has a $k$-partition, $\left\{F_{1}, \ldots, F_{k}\right\}$, with conv $F_{i} \subseteq T$ for $i=1, \ldots, k$. Then $\gamma(T) \leq k$, i.e., $T$ is a union of $k$ or fewer convex sets.

Let $F$ be a finite subset of $X$. We wish to show that $F$ has a 3-partition, $\left\{F_{1}, F_{2}, F_{3}\right\}$, with conv $F_{i} \subseteq X$ for $i=1,2,3$. We intend to construct a set $H$ such that: $\alpha(H) \leq$ 2, $\mathrm{cl} H$ is polygonal, $F \subset H \subset X, \operatorname{cl} H \backslash H \subset \operatorname{bd} \mathrm{cl} H$ and $\operatorname{dim} \operatorname{ker} \mathrm{cl} H=2$. A representation of $H$ as a union of three convex sets will imply, in particular, that $F$ has a partition as required. Thus, in order to complete our proof, it will suffice to deal with sets $X$ for which $S=\operatorname{cl}(X)$ is polygonal. In Theorem 9.1 below we construct a closed polygonal set $P$. We then define $H=P \cap X$ and show that $H$ satisfies the conditions above. Before embarking on the construction of $P$, we pause to discuss the important notions of relative convexity and relative convex hull.

Let $S$ be a subset of $\mathbb{R}^{d}$, or, for that matter, of any real vector space. (Here we do not necessarily assume that $S$ is closed.) We say that a subset $C$ of $S$ is convex relative to $S$ if, for any two points $x, y \in C,[x, y] \subset S$, implies $[x, y] \subset C$. The set $S$ itself, or the intersection of $S$ with any convex set, are examples of relatively convex subsets of $S$.

The intersection of any family of relatively convex subsets of $S$ is again convex relative to $S$. If $F$ is any (finite or infinite) subset of $S$, then the intersection of all relatively convex subsets of $S$ that include $F$ is clearly the smallest relatively convex subset of $S$ that includes $F$. It is called the relative convex hull of $F$ (relative to $S$ ). This relative convex hull can also be defined constructively, as follows: Put $F_{0}=F$. Define inductively, for $n \in \mathbb{N}, F_{n}$ to be the union of $F_{n-1}$ and all closed line segments $[x, y]$, where $x, y \in F_{n-1}$ and $[x, y] \subset S$. Then the relative convex hull of $F$ (relative to $S$ ) is the union $\cup_{n=0}^{\infty} F_{n}$. In many important cases this construction ends after a finite number of steps, i.e., $F_{n+1}=F_{n}$ for some finite $n$. 
Finally, let us note that if $C$ is a relatively convex subset of $S$, then $\alpha(C) \leq$ $\alpha(S), \beta(C) \leq \beta(S)$ and $\gamma(C) \leq \gamma(S)$. Reasons:

$\alpha$ : If $F \subset C$ is a visually independent subset of $C$, then $F$ is visually independent in $S$ as well.

$\beta$ : The invisibility graph of $C$ is a (spanned) subgraph of the invisibility graph of $S$. $\gamma$ : If $A$ is a convex subset of $S$, then $A \cap C$ is a convex subset of $C$.

Now we are ready to formulate Theorem 9.1:

Theorem 9.1 Suppose $S$ is a closed subset of $\mathbb{R}^{2}, \alpha(S) \leq 2, \underline{0} \in \operatorname{int} K(K=$ ker $S)$, and $F$ is a finite subset of $S$. Then there exists a set $P$ such that:

(1) $F \subset P \subset S$;

(2) $P$ is convex relative to $S$ (hence $\alpha(P) \leq 2$ );

(3) $\underline{0} \in$ int $\operatorname{ker} P$;

(4) $P$ is polygonal, i.e., $P$ consists of a simple closed polygonal line bd $P$ and its interior.

Proof We construct the set $P$ in several steps:

Step 1: Add to $F$ the origin $\underline{0}$, and, if necessary, a few more points of $S$ (never more than three), so as to make the origin $\underline{0}$ an interior point of the convex hull of the resulting set. Call the resulting set $F_{1}$.

Step 2: Define $S_{1}=S \cap \operatorname{conv} F_{1} . S_{1}$ satisfies all our assumptions on $S$, and is, in addition, compact. Proceed with $S$ replaced by $S_{1}$.

Step 3: Replace each point $a \in F_{1} \backslash\{\underline{0}\}$ by the intersection of $S_{1}$ with the closed ray $\{\lambda a: \lambda \geq 0\}$. Denote the resulting "sun" (union of segments emanating from $\underline{0}$ ) by $G$. The polygonal set $P$ promised in the theorem will be the convex hull of the "sun" $G$ relative to $S_{1}$ (or to $S$, does not matter).

Step 4: Now we start to construct the convex hull of $G$ relative to $S$. Assume $G=$ $\cup_{i=0}^{n-1}\left[\underline{0}, a_{i}\right]$, where the points $a_{i}$ are arranged in order of increasing argument. Define $a_{n}=a_{0}$ and denote by $\Delta_{i}(i=1,2, \ldots, n)$ the triangle $\left[\underline{0}, a_{i-1}, a_{i}\right]$. For each $i$, $1 \leq i \leq n$, we define a subset $P_{i}$ of $\Delta_{i}$ as follows:

Define

$$
\begin{aligned}
\lambda^{*} & =\max \left\{\lambda: 0 \leq \lambda \leq 1 \wedge\left[\lambda a_{i-1}, a_{i}\right] \subset S\right\} \\
\mu^{*} & =\max \left\{\mu: 0 \leq \mu \leq 1 \wedge\left[a_{i-1}, \mu a_{i}\right] \subset S\right\}
\end{aligned}
$$

The maxima do exist, since $S$ is closed.

Define $P_{i}=\left[\underline{0}, \lambda^{*} a_{i-1}, a_{i}\right] \cup\left[\underline{0}, a_{i-1}, \mu^{*} a_{i}\right] \subset \Delta_{i}$. If $\left[a_{i-1}, a_{i}\right] \subset S$, then $\lambda^{*}=$ $\mu^{*}=1$ and $P_{i}=\Delta_{i}$. If not, then $0<\lambda^{*}<1$ and $0<\mu^{*}<1$. ( $\lambda^{*}$ and $\mu^{*}$ are strictly positive, since an initial subinterval of $\left[\underline{0}, a_{i-1}\right]$ (and of $\left[\underline{0}, a_{i}\right]$ ) lies in $\operatorname{ker} S$ ). In this case, the intervals $\left[\lambda^{*} a_{i-1}, a_{i}\right]$ and $\left[a_{i-1}, \mu^{*} a_{i}\right]$ cross at some point $w_{i} \in$ int $\Delta_{i}$, and we obtain: $P_{i}=\left[\underline{0}, a_{i-1}, w_{i}\right] \cup\left[\underline{0}, a_{i}, w_{i}\right]$ (see Fig. 4). 
Fig. 4 Construction of $P_{i}$
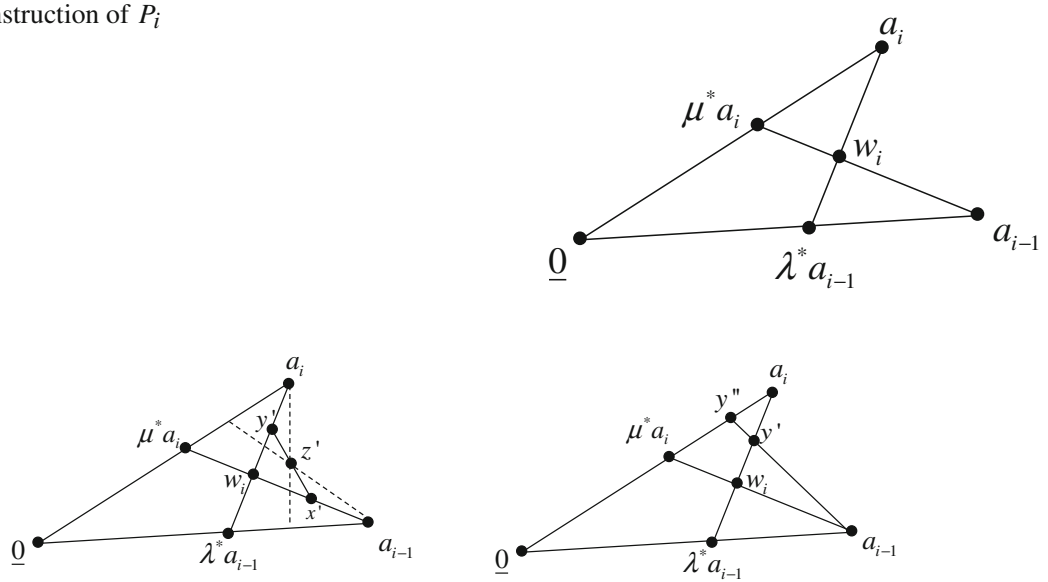

Fig. $5 P_{i}$ is convex relative to $\mathrm{S}$

Claim 9.2 The set $P_{i}$ is convex relative to $S$.

Proof This is obvious when $P_{i}=\Delta_{i}$. Assume, therefore, that $P_{i} \neq \Delta_{i}$, i.e., $P_{i}=$ $\left[\underline{0}, a_{i-1}, w_{i}\right] \cup\left[\underline{0}, a_{i}, w_{i}\right]=\Delta_{i} \backslash\left(\operatorname{int}\left[a_{i-1}, a_{i}, w_{i}\right] \cup\left(a_{i-1}, a_{i}\right)\right)$. Suppose, on the contrary, that some two points $x, y \in P_{i}$ see each other via $S$, but not via $P_{i}$. It follows that, say, $x \in\left[\underline{0}, a_{i-1}, w_{i}\right], y \in\left[\underline{0}, a_{i}, w_{i}\right]$, and the segment $[x, y]$ passes through $\Delta_{i} \backslash P_{i}\left(=\operatorname{int}\left[w_{i}, a_{i-1}, a_{i}\right] \cup\left(a_{i-1}, a_{i}\right)\right)$.

The segment $[x, y]$ cannot meet $\left(a_{i-1}, a_{i}\right)$, unless $x=a_{i-1}$ and $y=a_{i}$, in which case $P_{i}=\Delta_{i}$, contrary to our assumption. It follows that the segment $[x, y]$ crosses $\left[w_{i}, a_{i-1}\right]$ at some point $x^{\prime} \neq w_{i}$ and $\left[w_{i}, a_{i}\right]$ at some point $y^{\prime} \neq w_{i}$. If $x=x^{\prime}=a_{i-1}$ and $y^{\prime} \neq a_{i}$, extend the segment $\left[x, y^{\prime}\right]$ beyond $y^{\prime}$ into $P_{i}$, until it hits $\left[\underline{0}, a_{i}\right]$ at some point $y^{\prime \prime}$ (see the right side of Fig. 5). We find that $y^{\prime \prime}=\mu a_{i}$ for some $\mu^{*}<\mu<1$, but $a_{i-1}$ does see $y^{\prime \prime}$ via $S$, contrary to our definition of $\mu^{*}$. We obtain the same type of contradiction when $y=y^{\prime}=a_{i}$ but $x^{\prime} \neq a_{i-1}$.

Now suppose $y^{\prime} \neq a_{i}, x^{\prime} \neq a_{i-1}$. In this case $x^{\prime} \in\left(w_{i}, a_{i-1}\right), y^{\prime} \in\left(w_{i}, a_{i}\right)$. Put $z=1 / 2\left(x^{\prime}+y^{\prime}\right)$. If $a_{i-1}$ sees $z$ via $S$, then it sees via $S$ some point beyond $\mu^{*} a_{i}$ on $\left[\underline{0}, a_{i}\right]$, which is impossible. (Note that $S \cap \Delta_{i}$ is starshaped with respect to $\underline{0}$ )

We conclude that $\left[a_{i-1}, z\right] \nsubseteq S$. By the same token, $\left[a_{i}, z\right] \nsubseteq S$. But $\left[a_{i-1}, a_{i}\right] \nsubseteq$ $S_{1}$, as well, since $P_{i} \neq \Delta_{i}$. This contradicts our assumption that $\alpha(S) \leq 2$ (see the left side of Fig. 5).

dummy

Step 5: Define $P=\cup_{i=1}^{n} P_{i}$. Let us check that $P$ satisfies the requirements of Theorem 9.1.

By our construction, $F \subset F_{1} \subset G \subset P \subset S_{1} \subset S$.

To prove that $P$ is convex relative to $S$, we take two points $x, y \in P$ that see each other via $S$, and show that $[x, y] \subset P$.

If $x$ and $y$ belong to the same part $P_{i}$, then $[x, y] \subset P_{i} \subset P$, by Claim 9.2.

If $\underline{0} \in[x, y]$, then $[x, y] \subset P$, since $P$ is starshaped with respect to $\underline{0}$. 
Assume, therefore, that $x \in P_{i}$ and $y \in P_{j}, i<j$, and that the line through $x, y$ does not pass through the origin. Note that both $x$ and $y$ lie in $S_{1}\left(=S \cap \operatorname{conv} F_{1}\right)$ and, therefore, $[x, y] \subset S$ implies $[x, y] \subset S_{1}$.

For $v=0,1, \ldots, n$, denote by $R_{v}$ the ray emanating from $\underline{0}$ through $a_{v}\left(R_{v}=\right.$ $\left\{\lambda a_{v}: \lambda \geq 0\right\}$ ). The segment $[x, y]$ crosses the rays $R_{i}, R_{i+1}, \ldots, R_{j-1}$ (or $\left.R_{j}, R_{j+1}, \ldots, R_{n}, R_{1}, \ldots, R_{i-1}\right)$ in this order. Assume, for the sake of simpler notation, that it crosses $R_{i}, R_{i+1}, \ldots, R_{j-1}$.

Assume that $[x, y]$ meets $R_{v}$ at the point $b_{v}=\lambda_{v} a_{v}$, where $\lambda_{v}>0$. If $\lambda_{v}>1$ then $b_{v} \notin S_{1}$, since $a_{v}$ is the last point of $S_{1}$ on $R_{v}$. It follows that $0<\lambda_{v} \leq 1$, and, therefore, $b_{v} \in S_{1}$, hence $b_{v} \in P_{v} \cap P_{v+1}$ for $v=i, i+1, \ldots, j-1$. Thus $[x, y]=\left[x, b_{i}\right] \cup\left[b_{i}, b_{i+1}\right] \cup \cdots \cup\left[b_{j-2}, b_{j-1}\right] \cup\left[b_{j-1}, y\right]$. By Claim 9.2, $\left[x, b_{i}\right] \subset$ $P_{i},\left[b_{j-1}, y\right] \subset P_{j}$ and $\left[b_{v-1}, b_{v}\right] \subset P_{v}$ for $i<v<j$, hence $[x, y] \subset P$.

To show that $\underline{0} \in \operatorname{int} \operatorname{ker} P$, note that $\underline{0} \in \operatorname{int} \operatorname{ker} S$ and $\underline{0} \in$ int $P$. Let $U$ be a neighborhood of $\underline{0}$ that lies in $P \cap \operatorname{ker} S$. Every point $u \in U$ sees every point $p \in P(\subset S)$ via $S$, and, therefore, via $P$, since $P$ is convex relative to $S$.

Finally, note that the number of edges of the boundary of $P$ never exceeds $2\left|F_{1}\right|$.

We can now define the set $H$ as follows: $H=P \cap X$. Let us show that $H$ satisfies our requirements: (Recall that we need $H$ such that: $F \subset H \subset X, \alpha(H) \leq 2, \operatorname{cl} H$ is polygonal, $\operatorname{cl} H \backslash H \subset \operatorname{bdcl} H$ and $\operatorname{dimkercl} H=2$.)

According to our construction, $F \subset H \subset X$.

Let us show that $H$ is convex relative to $X$ : Take two points $a, b \in H$ such that $[a, b] \subset X . a, b \in P,[a, b] \subset S$, so, since $P$ is convex relative to $S,[a, b] \subset P$. Hence, $[a, b] \subset X \cap P=H$. Therefore, $\alpha(H) \leq 2$.

int $P \subset$ int $S \subset X$, so int $P \subset H=P \cap X$. Since $P=$ clint $P$, we find that cl $H=P$ is polygonal, and $\mathrm{cl} H \backslash H \subset P \backslash$ int $P=\operatorname{bd} P=\operatorname{bdcl} H$.

Finally, $\underline{0} \in$ int $\operatorname{ker} P$, so $\operatorname{dimkercl} H=2$. This concludes the reduction to the polygonal case. Therefore, we may assume that $S=\operatorname{cl} X$ is polygonal.

\section{Proof of Theorem E, The Polygonal Case. Preliminaries}

$S$ is a compact polygonal set. Denote by $Q$ the set of lnc points of S. Let $q_{1}, \ldots, q_{n}$ be the points of $Q$ ordered in clockwise direction along bd(conv $Q$ ). We assume, for the moment, that $n \geq 3$. The simpler cases $n=0,1,2$ will be considered afterward. conv $Q$ is a polygon with vertices $q_{1}, \ldots, q_{n}$ and edges $e_{i}=\left[q_{i}, q_{i+1}\right], i=1, \ldots, n$ (where $q_{n+1}=q_{1}$ ). By $e_{i_{+}}$we denote the closed half-plane determined by aff $e_{i}$ that misses intconv $Q$. According to Valentine's proof [6], $S$ is the union of conv $Q$ and $n$ "bumps" $W_{1}, \ldots, W_{n}$, where $W_{i}=S \cap e_{i_{+}}$. We shall refer to $W_{1}, \ldots, W_{n}$ as the leaves of $S$. Each $W_{i}$ is a convex polygon and so is the union $W_{i} \cup \operatorname{conv} Q$, for $i=1, \ldots, n$. Actually, the union of conv $Q$ with any set of leaves not containing two adjacent leaves, is a convex polygon.

If we orient the boundary of $S$ clockwise, the boundary of each leaf $W_{i}$ (excluding the base edge $e_{i}$ ) becomes a directed polygonal path, with a first edge starting at $q_{i}$ and a last edge ending at $q_{i+1}$. Take $l_{i}$ to be the line spanned by the last edge of $W_{i-1}$, and $m_{i}$ to be the line spanned by the first edge of $W_{i+1}$. Notice that if $\alpha, \beta, \gamma$ are the angles subtended by $W_{i-1}$, conv $Q$ and $W_{i}$ at $q_{i}$, as in Fig. 6, then the following holds: 
Fig. 6 A description of S

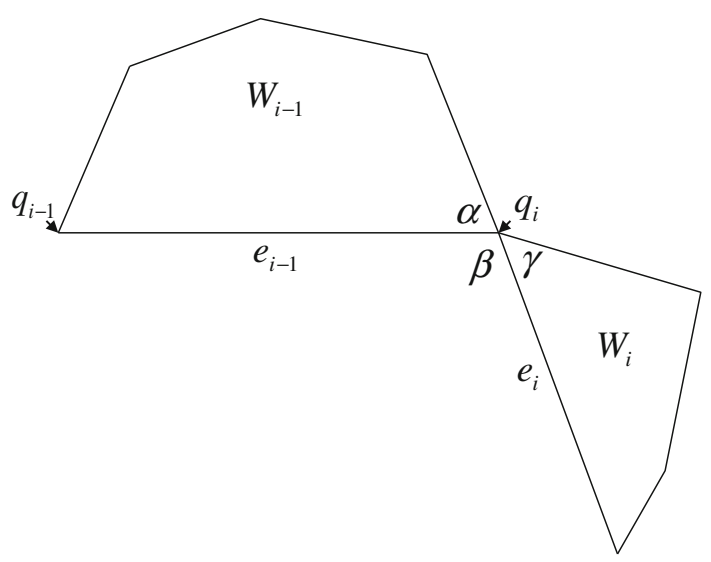

Fig. 7 A description of $\mathrm{X}$

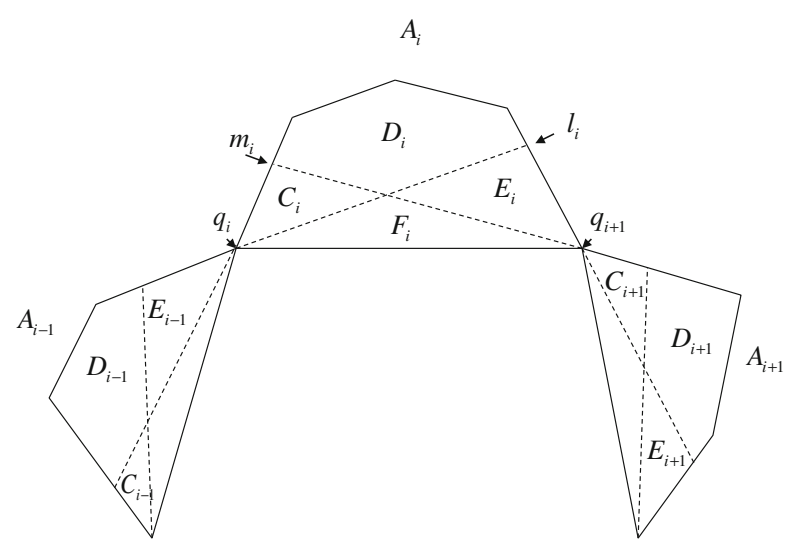

$\alpha+\beta \leq 180^{\circ}, \quad \beta+\gamma \leq 180^{\circ}$ and $\alpha+\beta+\gamma>180^{\circ}$. Therefore, $l_{i}$ passes either through int $W_{i}$ or through the basis $e_{i}$. The same holds for $m_{i}$ (see Fig. 7).

Denote by $l_{i_{+}}$the closed half-plane determined by $l_{i}$, that misses intconv $Q$ and by $m_{i_{+}}$the closed half-plane determined by $m_{i}$, that misses intconv $Q$.

Done with the description of $S$, we move on to describe $X$ : Define for $i=$ $1, \ldots, n, A_{i}=\left(W_{i} \cap X\right) \backslash Q$. These are the "leaves" of $X$. (Note that $A_{i}$ includes the relative interior of $e_{i}$, but not its endpoints $q_{i}, q_{i+1}$.) Now, since int $S \subset X, X$ can be represented as the following disjoint union: $X=$ int conv $Q \cup\left(\cup_{i=1}^{n} A_{i}\right) \cup(Q \cap X)$.

Before entering into more technicalities, we would like to give the reader an idea of how we properly color $X$ with three colors.

In the original proof of Valentine's Theorem (for $S$ ), each leaf $W_{i}$ (or, more precisely, $\left.W_{i} \backslash e_{i}\right)$, is colored uniformly, and two adjacent leaves get different colors. The central part, $\operatorname{conv} Q$, is part of $\operatorname{ker} S$, and need not be colored at all. Thus, two colors suffice locally, and the third color is only needed to close the circuit when $n$ is odd.

Passing to $X$, the set $A_{i}\left(=X \cap W_{i} \backslash Q\right)$ may miss some boundary points of $W_{i}$, and fail to be convex. This necessitates more than one color for $A_{i}$. We pass through each leaf $A_{i}($ of $X)$ the line $l_{i}$, that divides $A_{i}$ into an upper left part $\mathrm{NE}\left(C_{i} \cup D_{i}\right.$ in Fig. 7) 
and a lower right part SW $\left(F_{i} \cup E_{i}\right.$ in Fig. 7). The precise definition of this partition (i.e., which part includes $A_{i} \cap l_{i}$ ) will be given below.

The NE part is convex, and consists precisely of all points $x \in A_{i}$ that fail to see via $X$ some points in $A_{i-1}$. The SW part is also convex, except (possibly) for some local invisibilities on the boundary.

We color each of these two parts (NE and SW) uniformly with different colors. We also have to keep in mind that the color assigned to NE should be different from the colors assigned to the adjacent leaf $A_{i-1}$.

Such a coloring will also take care of at least part of the invisibilities along the boundary of $A_{i}$. If there is some invisibility left within the SW part (this can happen only if the lines $l_{i}, m_{i}$ do not cross within $A_{i}$ ), then we fix the coloring along the boundary using the third color (see the set $G_{i}$ below).

We can play the same trick with the line $m_{i}$, coming from the right, instead of $l_{i}$, but we shall not use this option. The following point, however, is important: Whenever the line $l_{i}$, or $m_{i}$, happens to be "horizontal," i.e., coincides with aff $e_{i}$, the leaf $A_{i}$ will be convex, and we may color it uniformly.

After having colored the $A_{i}$ 's, we finish the job by coloring $Q$. Each point $q_{i} \in Q$ belongs to $\operatorname{ker} S$, and sees via int $S$ (hence via $X$ ) almost all of $X . q_{i}$ may fail to see via $X$ only points that lie on the last edge of $A_{i-1}$ or $A_{i-2}$, or on the first edge of $A_{i}$ or $A_{i+1}$ (actually, on at most two of these edges simultaneously). Here the term "first (last) edge of $A_{i}$ " should be understood as "the intersection of $A_{i}$ with the first (last) edge of $W_{i}$." We shall see to it that the points that $q_{i}$ does not see use at most two colors, so there is a third color left for $q_{i}$.

At this point we start the precise, technical description of the promised 3-coloring of $X$. First, we define two partitions of $A_{i}$ into two parts:

$$
\begin{aligned}
& l_{i(+)}=\left\{\begin{array}{ll}
A_{i} \cap \operatorname{int}\left(l_{i_{+}}\right) & \text {if } X \cap l_{i} \text { isconvex, } \\
A_{i} \cap l_{i_{+}} & \text {otherwise. }
\end{array} \quad l_{i_{(-)}}=A_{i} \backslash l_{i_{(+)}},\right. \\
& m_{i_{(+)}}=\left\{\begin{array}{ll}
A_{i} \cap \operatorname{int}\left(m_{i_{+}}\right) & \text {if } X \cap m_{i} \text { isconvex, } \\
A_{i} \cap m_{i_{+}} & \text {otherwise. }
\end{array} \quad m_{i_{(-)}}=A_{i} \backslash m_{i_{(+)}} .\right.
\end{aligned}
$$

We shall now see that $l_{i_{(+)}}$is convex:

If $X \cap l_{i}$ is not convex then $l_{i_{(+)}}$includes $l_{i} \cap A_{i}$ and there is a point in the last edge of $W_{i-1}$ which is in $X$ and does not see any point in $l_{i_{(+)}}$, so due to $\alpha(X)=2$, every two points in $l_{i_{(+)}}$see each other via $l_{i_{(+)}}$. Otherwise, if $X \cap l_{i}$ is convex, then $l_{i_{(+)}}$does not include $l_{i} \cap A_{i}$, so for any two points $a, b$ in $l_{i_{(+)}}$there is a point in $A_{i-1}$ (close enough to the last edge of $W_{i-1}$ ) which sees neither $a$ nor $b$, hence $[a, b] \subset l_{i_{(+)}}$. Similarly, $m_{i_{(+)}}$is convex as well (Fig. 7).

It is easy to see that any point in $l_{i_{(-)}}$sees all points in $A_{i-1}$ via $X$. (The union $U_{i}=W_{i-1} \cup$ conv $Q \cup\left(l_{i_{-}} \cap W_{i}\right)$ is locally convex, and, therefore, a convex polygon, by Tietze's Theorem. Since int $S \subset X$, the only possible invisibilities in $X \cap U_{i}$ are along boundary edges of $U_{i}$. The edge determined by $l_{i}$ is taken care of by the exact definition of $l_{i_{(-)}}$. In case $n=3$ there may be another boundary edge of $U_{i}$ that reaches from $A_{i}$ to $A_{i-1}$, namely, the edge determined by the line aff $\left(q_{i+1}, q_{i+2}\right)$ 


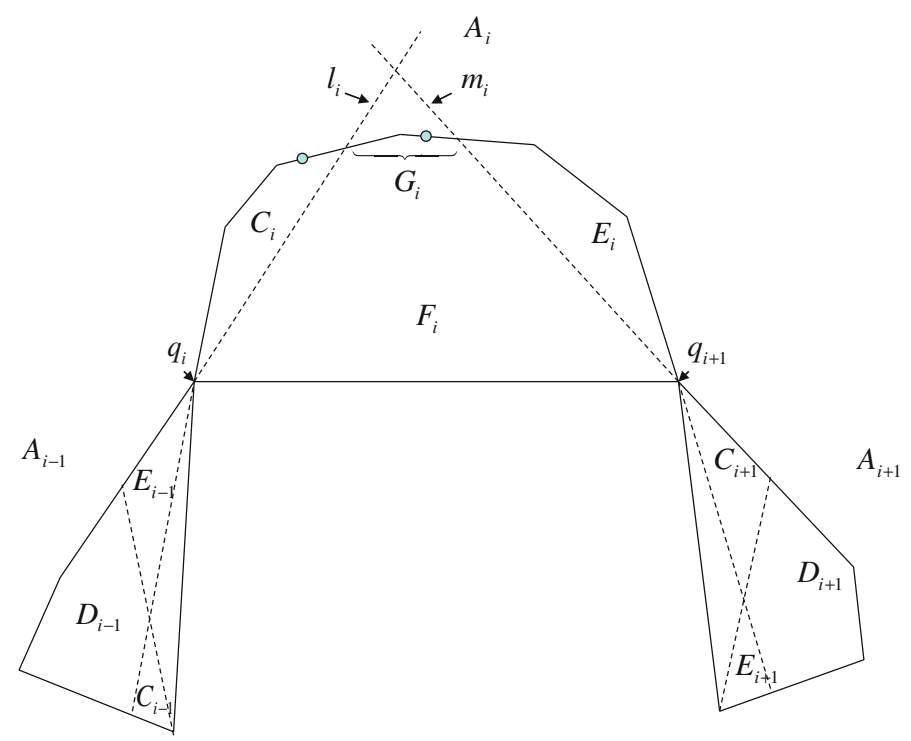

Fig. 8 Here $l_{i}$ and $M_{i}$ do not meet within $A_{i}$

(=aff $\left(q_{i+1}, q_{i-1}\right)$.) If this edge contains a point $x \in A_{i}$ and a point $y \in A_{i-1}$ then both $x$ and $y$ fail to see via $X$ any point $z \in$ int $W_{i+1}$, and, therefore, $x$ sees $y$ via $X$.) Similarly, any point in $m_{i_{(-)}}$sees all points in $A_{i+1}$ via $X$.

Now define:

$$
\begin{aligned}
D_{i} & =l_{i_{(+)}} \cap m_{i_{(+)}}, \\
C_{i} & =l_{i_{(+)}} \cap m_{i_{(-)}}, \\
E_{i} & =l_{i_{(-)}} \cap m_{i_{(+)}}, \\
F_{i} & =l_{i_{(-)}} \cap m_{i_{(-)}} \cap \operatorname{ker} X, \\
G_{i} & =l_{i_{(-)}} \cap m_{i_{(-)}} \backslash \operatorname{ker} X \text { (see Fig. 8). }
\end{aligned}
$$

Notice that Fig. 8 describes the case where $l_{i}, m_{i}$ meet in int $A_{i}$ (then $D_{i} \neq \phi$ and $G_{i}=\phi$ ). $G_{i}$ may be non-empty when $l_{i}, m_{i}$ do not meet within $A_{i}$ (as in Fig. 11), or even when they meet on the boundary of $A_{i}$.

\section{Proof of Theorem E, The Polygonal Case. Continued}

Stage 2 The requirements: As we are going to color each of $C_{i} \cup D_{i}, E_{i}$ uniformly, we will first see why each of them is convex:

Recall that $D_{i}=l_{i_{(+)}} \cap m_{i_{(+)}}, C_{i}=l_{i_{(+)}} \cap m_{i_{(-)}}$. So $C_{i} \cup D_{i}=l_{i_{(+)}}$, which is convex. Now, $E_{i}=l_{i_{(-)}} \cap m_{i_{(+)}}$so either $E_{i}=l_{i_{-}} \cap m_{i_{(+)}}$or $E_{i}=\operatorname{int}\left(l_{i_{-}}\right) \cap m_{i_{(+)}}$. In any case, $E_{i}$ is convex, as the intersection of two convex sets (Fig. 8). 
Next, we need to check what are other requirements there are for a coloring $c$ : $X \rightarrow\{0,1,2\}:$

Within each leaf $A_{i}: F_{i} \subset \operatorname{ker}(X)$, hence can be given any color. It is left to check the requirements for its complement in $A_{i}$. Since $W_{i}$ is convex, and int $W_{i} \subset A_{i}$, invisibility within $A_{i}$ can occur only along edges of $W_{i}$. Indeed, two points $a, b \in A_{i}$ do not see each other via $X$ iff:

(i) Both $a$ and $b$ belong to an edge $e$ of $W_{i}$ (not the base edge $\left[q_{i}, q_{i+1}\right]$, of course),

(ii) the intersection $e \cap A_{i}$ is not convex, and

(iii) $a, b$ belong to different components of $e \cap A_{i}$.

If $l_{i}$ and $m_{i}$ cross in int $W_{i}$, then invisibility within $A_{i}$ can be along at most one edge $e$ of $W_{i}$, that goes all the way from $C_{i}$ to $E_{i}$, with one component of $e \cap A_{i}$ in $C_{i}$, and the other in $E_{i}$. Our coloring will take care of this invisibility if we require:

Requirement $1 c\left(C_{i} \cup D_{i}\right) \neq c\left(E_{i}\right) \quad$ If $l_{i}$ and $m_{i}$ do not cross in int $W_{i}$, then invisibility within $A_{i}$ can occur within edges of $W_{i}$ that are not entirely confined to $C_{i}$ or to $E_{i}$, i.e., edges that cross from $C_{i}$ to $l_{\left.i_{(}\right)} \cap m_{i_{(-)}}$or lie entirely in $l_{\left.i_{(}\right)} \cap m_{i_{(-)}}$, or cross from $l_{i_{(-)}} \cap m_{i_{(-)}}$to $E_{i}$ (or, possibly, a single edge that reaches from $C_{i}$ through $l_{i_{(-)}} \cap m_{i_{(-}}$) all the way to $\left.E_{i}\right)$. $G_{i}$ consists of all points $a \in A_{i}$ that belong to $l_{i_{(-)}} \cap m_{i_{(-)}}$and fail to see some other points $b \in A_{i}$.

A detailed recipe for a 3-coloring that takes care of all these invisibilities is given in Stage 3.

Between two adjacent leaves Two points in adjacent leaves, $a \in A_{i}, b \in A_{i+1}$, may not see each other. This can happen only if $a \in m_{i_{(+)}}=D_{i} \cup E_{i}$ and $b \in l_{i+1_{(+)}}=$ $C_{i+1} \cup D_{i+1}$. Therefore, we require:

Requirement 2 For each $i, c\left(E_{i}\right) \neq c\left(C_{i+1} \cup D_{i+1}\right)$ and $c\left(C_{i} \cup D_{i}\right) \neq c\left(C_{i+1} \cup\right.$ $\left.D_{i+1}\right)$, where the addition of the indices is modulo $n$, i.e., $n+1=1$.

\section{Involvement of an lnc point $q_{i}$}

$q_{i}$ may fail to see a point that is in one of the following locations: a point in the last edge of $A_{i-1}\left(\subset D_{i-1} \cup E_{i-1}\right)$, a point in the first edge of $A_{i}\left(\subset C_{i} \cup D_{i}\right)$, a point in the last edge of $A_{i-2}\left(\subset D_{i-2} \cup E_{i-2}\right)$ (this can happen only if $l_{i-1}=\operatorname{aff}\left(q_{i-1}, q_{i}\right)$, in which case $A_{i-1}$ is convex), or a point in the first edge of $A_{i+1}\left(\subset C_{i+1} \cup D_{i+1}\right)$ (this can happen only if $m_{i}=\operatorname{aff}\left(q_{i}, q_{i+1}\right)$, in which case $A_{i}$ is convex). Now assume that $q_{i}$ does not see two points $a, b \in X$ at two different locations. Since $\alpha(X)=2$, there are 3 cases that cannot occur: $a \in A_{i-1}, b \in A_{i}, a \in A_{i-2}, b \in A_{i-1}$ and $a \in A_{i}, b \in A_{i+1}$. This leaves three possible cases:

1. $a \in A_{i-2}, b \in A_{i+1}$

2. $a \in A_{i-2}, b \in A_{i}$

3. $a \in A_{i-1}, b \in A_{i+1}$.

Thus it is impossible that $q_{i}$ will not see points at three different locations. In Stage 3 we shall produce a coloring for $q_{i}$ that copes with all three possible cases.

Between two leaves that are not adjacent If a point $x \in A_{i-1}$ does not see a point $y \in A_{i+1}$, then $x$ is necessarily on the last edge of $W_{i-1}$ and $y$ is on the first edge of $W_{i+1}$, and these edges lie on the same line, i.e., $m_{i}=l_{i}=\operatorname{aff}\left(q_{i}, q_{i+1}\right)$. In this case, 
both $x$ and $y$ do not see any point of $A_{i}$. This leads to a contradiction to $\alpha(X)=2$. Therefore, invisibility is impossible among two leaves of $X$ which are two edges apart.

In any other case, for any two points $a, b \in X$ such that $a \in A_{i}, b \in A_{j}$ and $i+2<j<i+n-2$, the segment $(a, b)$ is in int $S$ (according to Valentine [6]), and, therefore, is in $X$.

\section{Proof of Theorem E, The Polygonal Case-Coloring}

Stage 3 A recipe for a coloring $c: X \rightarrow\{0,1,2\}$ : Since $\operatorname{int}(\operatorname{conv} Q) \subset \operatorname{ker} X$, we only need to color $\left(\cup_{i=1}^{n}\left(A_{i} \backslash F_{i}\right) \cup(Q \cap X)\right.$. We start by coloring $A_{i} \backslash F_{i}$ :

The coloring of $C_{i} \cup D_{i}, E_{i}$

General rule: $c\left(C_{i} \cup D_{i}\right) \equiv i(\bmod 3)$ and $c\left(E_{i}\right) \equiv i+2(\bmod 3)$ for all $1 \leq i \leq n$. Exceptions

- Case $0, n \equiv 0(\bmod 3)$ : No exceptions.

- Case $1, n \equiv 1(\bmod 3): c\left(C_{n} \cup D_{n}\right)=2$ and $c\left(E_{n-1}\right)=1$. See Fig. 9 for an example with $n=4$.

- Case $2, n \equiv 2(\bmod 3): c\left(E_{n}\right)=0$. See Fig. 10 for an example with $n=5$.

Let us now check that the proposed coloring does satisfy Requirements 1 and 2, namely, that

(a) $c\left(C_{i} \cup D_{i}\right) \neq c\left(E_{i}\right)$,

(b) $c\left(C_{i} \cup D_{i}\right) \neq c\left(C_{i+1} \cup D_{i+1}\right)$,

(c) $c\left(E_{i}\right) \neq c\left(C_{i+1} \cup D_{i+1}\right)$ for $i=1,2, \ldots, n$, where the addition of the indices is modulo $n$.

These conditions clearly hold in Case 0 (i.e., when $3 / n$ ). In Cases 1 and 2 we only have to check conditions (a), (b), and (c) in the instances where the definition of $c\left(C_{i} \cup D_{i}\right)$, or of $c\left(C_{i+1} \cup D_{i+1}\right)$, or of $c\left(E_{i}\right)$, is exceptional, and conditions (b) and

Fig. 9 Coloring of the

polygonal case where $n=4$

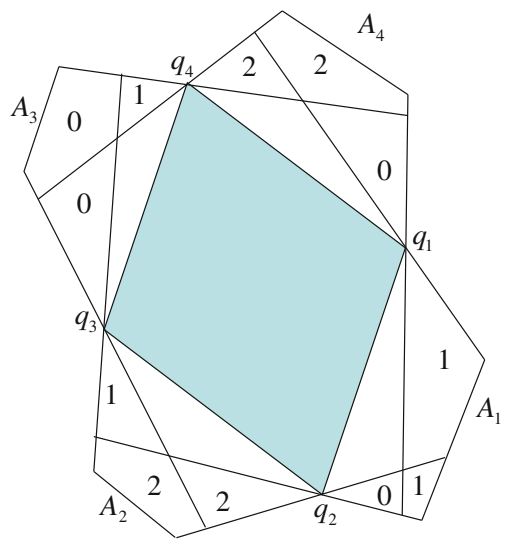


Fig. 10 Coloring of the polygonal case where $n=5$

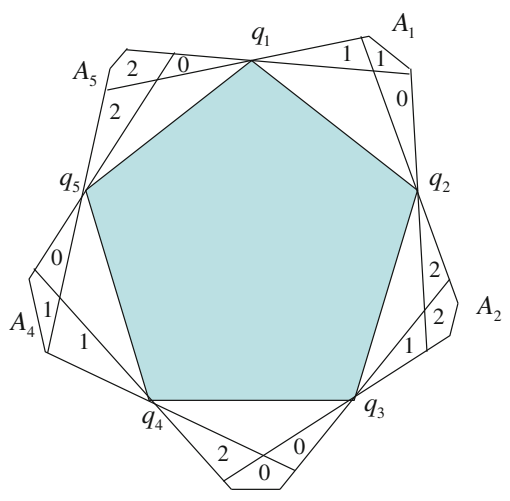

$A_{3}$

(c) for $i=n$, because of the "seam" modulo 3 between $i=n$ and $i+1=1$. Let us do this:

\section{Case 1}

(a) $c\left(C_{n-1} \cup D_{n-1}\right)=0 \neq 1=c\left(E_{n-1}\right), c\left(C_{n} \cup D_{n}\right)=2 \neq 0=c\left(E_{n}\right)$,

(b) $c\left(C_{n-1} \cup D_{n-1}\right)=0 \neq 2=c\left(C_{n} \cup D_{n}\right), c\left(C_{n} \cup D_{n}\right)=2 \neq 1=c\left(C_{1} \cup D_{1}\right)$,

(c) $c\left(E_{n-1}\right)=1 \neq 2=c\left(C_{n} \cup D_{n}\right), c\left(E_{n}\right)=0 \neq 1=c\left(C_{1} \cup D_{1}\right)$,

\section{Case 2}

(a) $c\left(C_{n} \cup D_{n}\right)=2 \neq 0=c\left(E_{n}\right)$,

(b) $c\left(C_{n} \cup D_{n}\right)=2 \neq 1=c\left(C_{1} \cup D_{1}\right)$,

(c) $c\left(E_{n}\right)=0 \neq 1=c\left(C_{1} \cup D_{1}\right)$.

(As a matter of fact, the check can be performed by a close look at Figs. 9 and 10.) The coloring of $q_{i} \in X$ :

As mentioned in Stage 2, there are three possible "maximal" cases where $q_{i}$ does not see via $X$ points $a, b$ at two different locations:

Case $1 a \in A_{i-2}, b \in A_{i+1}$ : In this case, $a \in D_{i-2} \cup E_{i-2}$ and $b \in C_{i+1} \cup D_{i+1}$. In addition, $a \in \operatorname{aff}\left(q_{i-1}, q_{i}\right), b \in \operatorname{aff}\left(q_{i}, q_{i+1}\right)$. It follows that Case 1 cannot occur for two adjacent lnc points $q_{i}, q_{i+1}$. Otherwise, if $q_{i}$ does not see via $X$ two points $a \in A_{i-2}, b \in A_{i+1}$ and $q_{i+1}$ does not see via $X$ two points $a^{\prime} \in A_{i-1}, b^{\prime} \in A_{i+2}$, then $a^{\prime} \in \operatorname{aff}\left(q_{i}, q_{i+1}\right), b \in \operatorname{aff}\left(q_{i}, q_{i+1}\right)$. Hence, the points $a^{\prime}, q_{i}, b$ do not see each other via $X$, a contradiction to $\alpha(X)=2$. Now we specify the color of $q_{i}$ in Case 1 , dealing with the three congruence classes modulo 3 separately.

If $n=0(\bmod 3)$ : According to the coloring $c$, in this case, for every $1 \leq i \leq$ $n, \quad c\left(C_{i+1} \cup D_{i+1}\right)=i+1(\bmod 3), c\left(C_{i-2} \cup D_{i-2}\right)=i-2(\bmod 3)$, therefore, $c\left(C_{i+1} \cup D_{i+1}\right)=c\left(C_{i-2} \cup D_{i-2}\right)$ so those two sets are colored by the same color. The set $E_{i-2}$ is colored by a second color. As these sets are the only constraints for $q_{i}$, we will color $q_{i}$ by the third color that is left "free."

If $n=1(\bmod 3)$ : For $3 \leq i \leq n-2$, the same considerations as in the case $n=0$ $(\bmod 3)$ hold. $\left(i=n-2\right.$ is not exceptional, since we make no use of $\left.E_{i+1}\right)$. 
For $i=n, c\left(C_{i+1} \cup D_{i+1}\right)=c\left(C_{1} \cup D_{1}\right)=1, c\left(C_{i-2} \cup D_{i-2}\right)=c\left(C_{n-2} \cup\right.$ $\left.D_{n-2}\right)=n-2(\bmod 3)=2, c\left(E_{i-2}\right)=c\left(E_{n-2}\right)=n(\bmod 3)=1$, so we define $c\left(q_{n}\right)=0$.

For $i=2, c\left(C_{i+1} \cup D_{i+1}\right)=c\left(C_{3} \cup D_{3}\right)=0, c\left(C_{i-2} \cup D_{i-2}\right)=c\left(C_{n} \cup D_{n}\right)=$ 2, $c\left(E_{i-2}\right)=c\left(E_{n}\right)=n+2(\bmod 3)=0$, so we define $c\left(q_{2}\right)=1$.

For $i=1$ or $i=n-1$, there is no color left to assign to $q_{i}$, since all three colors are used by $C_{i+1} \cup D_{i+1}, C_{i-2} \cup D_{i-2}$ and $E_{i-2}$. We cope with this situation by renumbering the lnc points in a way that Case 1 occurs neither in $q_{1}$, nor in $q_{n-1}$. This is certainly possible if Case 1 does not occur at all. If it does occur, mark one lnc point where it occurs by $q_{n}$, and recall that Case 1 cannot occur in two adjacent lnc points.

If $n=2(\bmod 3)$ : For $3 \leq i \leq n-1$, the same considerations as in the case $n=0$ $(\bmod 3)$ hold.

For $i=2, c\left(C_{i+1} \cup D_{i+1}\right)=c\left(C_{3} \cup D_{3}\right)=0, c\left(C_{i-2} \cup D_{i-2}\right)=c\left(C_{n} \cup D_{n}\right)=n$ $(\bmod 3)=2, c\left(E_{i-2}\right)=c\left(E_{n}\right)=0$, so we define $c\left(q_{2}\right)=1$.

For $i=1$ or $i=n$, there is no color left to assign to $q_{i}$, since all three colors are used by $C_{i+1} \cup D_{i+1}, C_{i-2} \cup D_{i-2}$, and $E_{i-2}$. If Case 1 does not occur in some two adjacent lnc points, then we renumber the lnc points in such a way that Case 1 occurs neither in $q_{1}$, nor in $q_{n}$. Notice that if $n$ is odd, then this must happen, since Case 1 cannot occur in two adjacent lnc points. If $n$ is even, then it may happen that Case 1 occurs in every second lnc point, but then all the sets $A_{i}$ are convex. In this special case we color $X$ differently: we color all $A_{i}$ 's alternately by two colors and the lnc points by the third color (see Fig. 11).

Case $2 a \in A_{i-2}, b \in A_{i}$ : Color $q_{i}$ by $c\left(C_{i-1} \cup D_{i-1}\right)$. Notice that $b \in C_{i} \cup D_{i}$. Now, $c\left(C_{i-1} \cup D_{i-1}\right) \neq c\left(C_{i} \cup D_{i}\right)$, so $q_{i}$ and $b$ are colored differently. Similarly, $c\left(C_{i-1} \cup D_{i-1}\right)$ differs from both $c\left(C_{i-2} \cup D_{i-2}\right)$ and $\left(E_{i-2}\right)$, so $q_{i}$ and $a$ are colored differently.

Case $3 a \in A_{i-1}, b \in A_{i+1}$ : Color $q_{i}$ by $c\left(C_{i} \cup D_{i}\right)$. Considerations similar to those in Case 2 show that $q_{i}$ is colored differently from both $a, b$.

The coloring proposed in Case 2 or in Case 3 clearly works also when $q_{i}$ fails to see points in only one (or none) of the leaves $A_{i-2}, A_{i-1}, A_{i}, A_{i+1}$.

Fig. 11 Coloring the lnc points

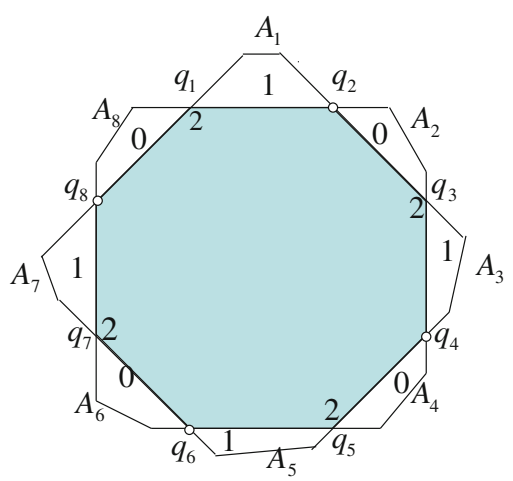


The coloring of $G_{i}: G_{i}$ is the disjoint union of finitely many connected components $b_{j}$. Each component is either a single point, or a line segment, or the union of two line segments with a common endpoint. (This common endpoint must, of course, be a vertex of $W_{i}$ ) Each edge of $W_{i}$ meets at most two components of $G_{i}$. If $G_{i} \neq \phi$, then $D_{i}=\phi$, and all the components $b_{j}$ lie in the gap between $C_{i}$ and $E_{i}$ (see Fig. 8). Number the components $b_{1}, \ldots, b_{t}$ in the order they appear on the boundary of $A_{i}$, with $b_{1}$ closest to $C_{i}$ and $b_{t}$ closest to $E_{i}$.

Points of $b_{1}$ may fail to see points of $C_{i}$, and points of $b_{t}$ may fail to see points of $E_{i}$. Beyond that, points of $G_{i}$ may fail to see each other via $X$ only if they belong to adjacent components $b_{j}, b_{j+1}$.

Assume $\{0,1,2\}=\{p, q, r\}$, where $p=c\left(E_{i}\right)$ and $q=c\left(C_{i}\right)$. Color the components $b_{j}$ as follows:

$$
c\left(b_{j}\right)=\left\{\begin{array}{l}
p \text { if } j \text { is odd and } j \neq t \\
q \text { if } j \text { is even, } \\
r \text { if } j \text { is odd and } j=t
\end{array}\right.
$$

Note that $c\left(b_{1}\right) \neq c\left(C_{i}\right), c\left(b_{t}\right) \neq c\left(E_{i}\right)$, and $c\left(b_{j}\right) \neq c\left(b_{j+1}\right)$ for $j=1,2, \ldots, t-$ 1. This finishes the description of a 3-coloring of $X$.

We still have to deal with the cases $n=0,1,2$.

\section{Proof of Theorem E, The Polygonal Case, Some Residual Cases}

Denote by $n(=|Q|)$ the number of lnc points of $S$. In the preceding section we gave a detailed proof of Theorem $\mathrm{E}$ under the assumption that $3 \leq n<\infty$. The cases $n=0$ (i.e., $S$ is convex), $n=1$ and $n=2$ are much simpler. The interested reader may find a detailed treatment of these cases under the same section number in the full version of this paper on the web (www.ma.huji.ac.il/ noanitzan/non-convexity.pdf, p. 36, Sect. 13).

This completes the proof that if $M_{i}=\phi$ and $\operatorname{dim} K=2$, then $\gamma(X) \leq 3$, and with it the proof of Theorem E.

Example 5 (due to Breen [1]) shows that the number three is the best possible.

Example 5 Let $P$ be a regular pentagon. Define $X=(P \backslash$ bd $P) \cup \operatorname{vert} P$ (see Fig. 12).

Fig. 12 An example with $M_{i}=\phi, \alpha=2$ and $\gamma=3$

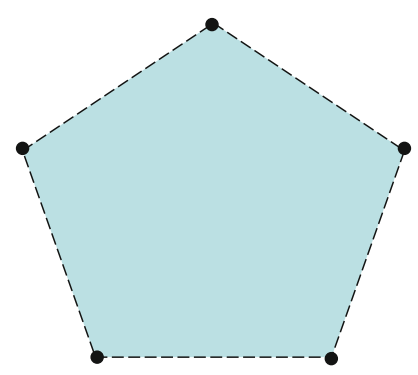


$\alpha(X)=2$ : The only points in $X$ that are not in $\operatorname{ker} X$ are the vertices of $P$. The only points that a vertex does not see via $X$ are the two adjacent vertices, but these two see each other via $X$.

There is a 5-circuit of invisibility, therefore, $\gamma(X) \geq 3$.

\section{Proof of Theorem $F$}

Assume $M_{i}=\{(0,0)\}$. Define $A=\left\{(x, y) \in \mathbb{R}^{2}: y>0 \vee(y=0 \wedge x>0)\right\}, B=$ $\left\{(x, y) \in \mathbb{R}^{2}: y<0 \vee(y=0 \wedge x<0)\right\} . A \cup B=\mathbb{R}^{2} \backslash\{(0,0)\}$ and $(0,0) \notin X$, therefore, $X=(X \cap A) \cup(X \cap B)$. $A, B$ are both convex, so each of the sets $X \cap A, X \cap B$ satisfies the conditions of Theorem $\mathrm{E}$, and, therefore, each is a union of three convex sets. Hence, $X$ is the union of six convex sets.

Example 6 shows that the number six is the best possible.

Example 6 We describe a set $X \subset \mathbb{R}^{2}$ and show that $\alpha(X)=2$ and $\gamma(X)>5$. Let $P$ be a regular 48 -gon with center $O$, vertices $p_{0}, p_{1}, \ldots, p_{48}\left(p_{0}=p_{48}\right)$ and edges $e_{i}=\left[p_{i-1}, p_{i}\right](i=1,2, \ldots, 48)$. Above each edge $e_{i}$ erect a triangular dome $T_{i}=\left[p_{i-1}, p_{i}, t_{i}\right]$. The interior angles of each $T_{i}$ are as follows:

At the odd-numbered base vertex: $7.5^{\circ}\left(=360^{\circ} / 48\right)$.

At the even-numbered base vertex: $6^{\circ}$.

At the tip $t_{i}: 166.5^{\circ}$.

Define $S=P \cup\left(\cup_{i=1}^{48} T_{i}\right) . X$ is obtained from $S$ by removing the odd-numbered vertices $p_{2 k-1}(k=1,2, \ldots 24)$ and the center $O$.

Note that each odd-numbered vertex $p_{2 k-1}$ is the crossing point of the segments $\left[p_{2 k-2}, t_{2 k}\right]$ and $\left[t_{2 k-1}, p_{2 k}\right]$. Moreover, the sum of the interior angles of $T_{2 k}, P$ and $T_{2 k+1}$ at the even-numbered vertex $p_{2 k}$ is $184.5^{\circ}\left(>180^{\circ}\right)$. Therefore, $t_{2 k}$ and $t_{2 k+1}$ do not see each other via $X$. Thus we see that, for each $k$, the points $\left\langle p_{2 k}, t_{2 k-1}, t_{2 k}, t_{2 k+1}, t_{2 k+2}, p_{2 k}\right\rangle$ form a 5-circuit of invisibility in $X$. Note also that $t_{i}$ and $t_{i+2}$ always see each other via int $X$ (see Fig. 13).

It can be easily shown that $\alpha(X)=2$. (This is shown in full in the detailed version: www.ma.huji.ac.il/ noanitzan/non-convexity.pdf , p. 42, Sect. 14.)

It is left to convince the reader that $\gamma(X)>5$. The 12 rays $\overrightarrow{O P_{4 k}}$

Fig. 13 Construction of an example with $\alpha=2, \gamma=6$

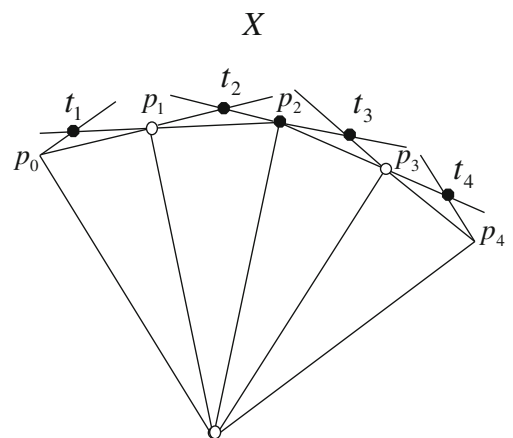


$(k=0, \ldots, 11)$ divide $X$ into twelve congruent sectors. Each sector includes four consecutive edges of $P$, and the corresponding domes, and contains a 5-circuit of invisibility. Figure 13 represents one sector. (The central angles are, of course, exaggerated.)

It follows that each sector is not a union of two convex sets, and, therefore, in any covering of $X$ by convex subsets, each sector will meet at least three of the covering subsets. Now, assume to the contrary that $X$ is the union of 5 convex sets. Let us try to evaluate the number of incidences between the five convex sets and the 12 sectors. On the one hand, as every sector meets at least three convex sets, this number is no less than $3 \cdot 12=36$. On the other hand, as none of the convex sets includes the center $O$, each convex set lies on one side of a line through $O$, and, therefore, meets at the most 7 sectors. Therefore, the number of incidences is not more than $7 \cdot 5=35$, a contradiction.

\section{Proof of Theorem G}

In the proof of Theorem E, in the case where $n=0$ (Sect. 13), we stated the following characterization: When $S$ is convex and $M_{i}=\phi, \gamma(X)=3$ iff $S$ is an odd-sided convex polygon (not a triangle), $X$ contains all vertices of $S$ and misses at least one point in each edge of $S$. (Otherwise $\gamma(X) \leq 2$.)

Assume $M_{i}=\{(0,0)\}$. Define $A$ and $B$ as in the proof of Theorem F (Sect. 14), to obtain $X=(X \cap A) \cup(X \cap B)$. $A, B$ and cl $X$ are all convex.

Let us show that the set $\operatorname{cl}(X \cap A)$ is convex. Since $(0,0) \in$ int $S$, we can choose a point $z=\left(x_{0}, y_{0}\right) \in$ int $S$ with $y_{0}>0$, i.e., $z \in$ int $S \subset X$. Now assume that $p, q \in \operatorname{cl}(X \cap A)(\subset \operatorname{cl} X \cap \operatorname{cl} A)$, and $r=(1-\lambda) p+\lambda q$ for some $0<\lambda<1$. We must show that $r \in \operatorname{cl}(X \cap A)$ as well. Define $p_{n}=\left(1-\frac{1}{n}\right) p+\frac{1}{n} z, q_{n}=\left(1-\frac{1}{n}\right) q+$ $\frac{1}{n} z, r_{n}=\left(1-\frac{1}{n}\right) r+\frac{1}{n} z$. Then $p_{n}, q_{n} \in \operatorname{int} A \cap \operatorname{int} S$ for all $n$. The sets $\operatorname{int} A$, int $S$ are convex. Therefore, for all $n, r_{n}=(1-\lambda) p_{n}+\lambda q_{n} \in \operatorname{int} A \cap \operatorname{int} S \subset A \cap X$, and $r=\lim _{n \rightarrow \infty} r_{n} \in \operatorname{cl}(A \cap X)$. By the same token, $\operatorname{cl}(X \cap B)$ is convex as well. Therefore, each of $X \cap A, X \cap B$ satisfies the conditions of the characterization brought above. Each of these sets has an edge with a missing vertex. Therefore, according to that characterization, each of $X \cap A, X \cap B$ is the union of at most two convex sets, hence $\gamma(X) \leq 4$.

Example 7 shows that the number four is the best possible.

Example 7 We describe a convex set $X \subset \mathbb{R}^{2}$ and show that $\alpha(X)=2$ and $\gamma(X) \geq 4$. Let $P$ be a regular 7-gon with center $O$. We define $X=P \backslash(\{O\} \cup \operatorname{bd} P) \cup \operatorname{vert} P$. ( $X$ is obtained from $P$ by removing the center $O$ and the relative interiors of all edges.) Let $C$ be a convex subset of $X$. Since $O \notin X, C$ is included in a closed half-plane $H$ with $O \in \operatorname{bd} H$. $H$ intersects vert $P$ in a stretch of three or four consecutive vertices. But two adjacent vertices of $X$ do not see each other via $X$. Therefore, $C$ contains at most two vertices of $P$. It follows that $\gamma(X) \geq 4$.

We show now that $\alpha(X)=2: X \backslash \operatorname{vert} P$ is the union of two convex sets. Therefore, if there is a 3 -circuit of invisibility in $X$, then it must contain a vertex of $P$. For each vertex $v$ of $P$, the points in $X$ that it does not see via $X$ are the two vertices adjacent to $v$ 
in $P$ and the opposite radius. Notice that all these points see each other via $X$. Therefore, a vertex of $P$ cannot participate in a 3-circuit of invisibility, hence $\alpha(X)=2$.

It is left to show that if $M_{\mathrm{b}}=\phi$ or $M_{\mathrm{b}}=$ bd $S$, then $\gamma(X)=2$. Indeed, if $M_{\mathrm{b}}=\phi$ then $X=S \backslash\{O\})=(S \cap A) \cup(S \cap B)$, where $A, B$ are the convex sets defined above. If $M_{\mathrm{b}}=$ bd $S$, then $X=$ int $S \backslash\{O\}=$ (int $\left.S \cap A\right) \cup$ (int $S \cap B$ ).

\section{Proof of Main Theorem 2}

If $\beta(X)=2$ then $\alpha(X)=2$. If $M_{i}=\phi$ then according to Theorem $\mathrm{E}, \gamma(X) \leq 3$. If $\left|M_{i}\right|>1$ then according to Theorem $\mathrm{C}, \gamma(X) \leq 3$. It is left to handle the case $\left|M_{i}\right|=1$ :

Assume $M_{i}=\{p\}$. We claim that for every point $x \in X,(p, x] \subset X$. Recall that $p \in$ int $S$ and that int $S \backslash\{p\} \subset X$. If $(p, x] \nsubseteq X$ for some $x \in X$, then, for some sufficiently small $\varepsilon>0$, the three points $x, p+\varepsilon(x-p), p-\varepsilon(x-p)$ are in $X$ and fail to see each other via $X$, and thus $\alpha(X)>2$.

Define $X^{*}=X \cup\{p\}$. The argument in the preceding paragraph shows that $p \in$ $\operatorname{ker} X^{*}$. It follows that a 2-coloring of the invisibility graph of $X$ can be extended to $X^{*}$ by assigning to $p$ either color. Therefore, $\beta\left(X^{*}\right) \leq 2$. Moreover, $\operatorname{cl}\left(X^{*}\right)=\operatorname{cl} X=S$, and thus $M_{i}\left(X^{*}\right)=\phi$. Hence, by Lemma 8.2, $\gamma\left(X^{*}\right)=\beta\left(X^{*}\right) \leq 2$. If $X^{*}$ is the union of two convex sets $A, B$, then $X=X^{*} \backslash\{p\}=(A \backslash\{p\}) \cup(B \backslash\{p\})$. Each of the sets $A \backslash\{p\}, B \backslash\{p\}$ is the union of at most two convex sets, and, therefore, $\gamma(X) \leq 4$.

Example 3 in Sect. 7 (a punctured Star of David) satisfies $\beta(X)=2$ and $\gamma(X)=4$. A proper 2-coloring is shown in the right part of Fig. 3.

\section{Example 8}

We describe a set $X \subset \mathbb{R}^{2}$ and show that $\alpha(X)=2$ and $\beta(X)=4$. Start with a square $A B C D$ topped by the upper half of a regular 16-gon with vertices $p_{-4}, p_{-3}, \ldots, p_{0}, \ldots, p_{3}, p_{4}\left(p_{-4}=D, p_{4}=C\right)$ and edges $e_{i}=\left[p_{i-1}, p_{i}\right], e_{-i}=$ $\left[p_{-i}, p_{-i+1}\right](i=1,2,3,4)$. Above each edge $e_{i}\left(e_{-i}\right)$ erect a triangular dome $T_{i}=\left[p_{i-1}, p_{i}, t_{i}\right] \quad\left(T_{-i}=\left[p_{-i}, p_{-i+1}, t_{-i}\right]\right), i=1,2,3,4$. The interior angles of each $T_{i}$ and $T_{-i}$ are as follows:

At the odd-numbered base vertex: $22.5^{\circ}$.

At the even-numbered base vertex: $18^{\circ}$.

At the tip $t_{i}\left(t_{-i}\right): 139.5^{\circ}$.

Define $S=\operatorname{conv}\left\{A, B, p_{-4}, \ldots, p_{0}, \ldots, p_{4}\right\} \cup \cup_{i=1}^{4}\left(T_{i} \cup T_{-i}\right) . X$ is obtained from $S$ by removing the odd-numbered vertices $p_{-3}, p_{-1}, p_{1}, p_{3}$, the center $O$ of the square $A B C D$ and the midpoint $q$ of the base $[A, B]$ (see Fig. 14).

$\alpha(X)=2$ : For the proof that $\alpha(X)=2$ we refer the reader to the detailed version (www.ma.huji.ac.il/ noanitzan/non-convexity.pdf, p. 46, Sect. 17). Here we only show that $\beta \geq 4$.

$\beta(X) \geq 4$ : Assume, on the contrary, that there exists a 3-coloring $c: X \rightarrow\{1,2,3\}$, such that any two points colored alike see each other via $X$. If $x \in[A, q)$ and $y \in(q, B]$ then $[x, y] \nsubseteq X$, and, therefore, $c(x) \neq c(y)$. It follows that one of the half-bases 
Fig. 14 An example with $\alpha=2, \beta=4$

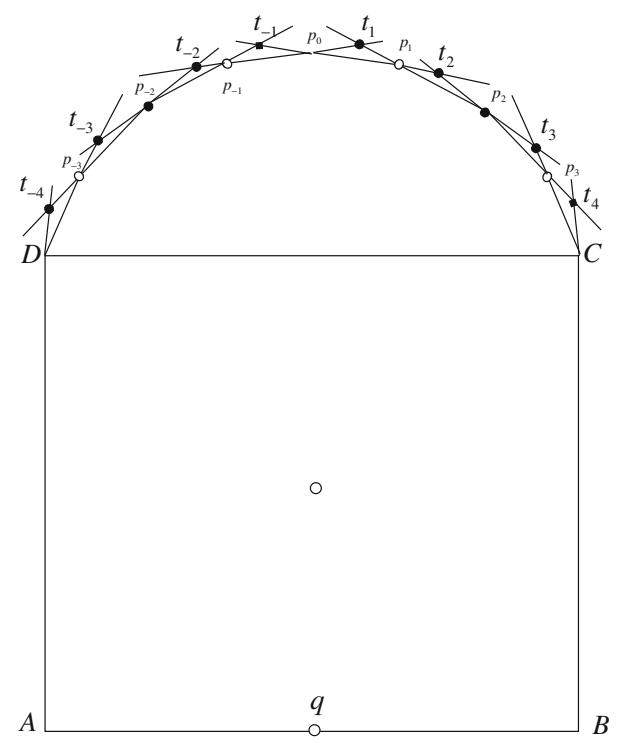

$[A, q),(q, B]$ (say $[A, q))$ is colored uniformly. But this leaves only two colors for the 5 -circuit of invisibility $\left\langle t_{1}, p_{2}, t_{4}, t_{3}, t_{2}\right\rangle$, in view of the missing center $O$.

$\beta(X) \leq 4$ : Use color 1 for the point $p_{-2}$ and for the segments $[A, D)$ and $[A, q)$, color 2 for the point $p_{2}$ and for the segments $[B, C)$ and $[B, q)$, color 3 for the intersection of $X \backslash\left\{p_{-2}, p_{0}, p_{2}\right\}$ with $T_{-4}, T_{-2}, T_{1}$ and $T_{3}$, and color 4 for the intersection of $X \backslash\left\{p_{-2}, p_{0}, p_{2}\right\}$ with $T_{-3}, T_{-1}, T_{2}$ and $T_{4}$. Now extend the coloring radially from $O$. Finally, use color 1 for the segments $\left(O, p_{-3}\right),\left(O, p_{-1}\right)$ and $\left(O, p_{0}\right]$, and color 2 for the segments $\left(O, p_{1}\right),\left(O, p_{3}\right)$ and $(O, q)$.

\section{References}

1. Breen, M.: Decomposition theorems for 3-convex subsets of the plane. Pac. J. Math. 53, 43-57 (1974)

2. Breen, M., Kay, D.: General decomposition theorems for $m$-convex sets in the plane. Israel J. Math. 24, 217-233 (1976)

3. Lawrence, J.F., Hare, W.R., Kenelly, J.W.: Finite unions of convex sets. Proc. Am. Math. Soc. 34, 225-228 (1972)

4. Matoušek, J., Valtr, P.: On visibility and covering by convex sets. Israel J. Math. 113, 341-379 (1999)

5. Perles, M.A., Shelah, S.: A closed $(\mathrm{n}+1)$-convex set in $\mathbb{R}^{2}$ is a union of $n^{6}$ convex sets. Israel J. Math. 70, 305-312 (1990)

6. Valentine, F.A.: A three point convexity property. Pac. J. Math. 7(2), 1227-1235 (1957)

7. Eggleston, H.G.: A condition for a compact plane set to be a union of finitely many convex sets. Proc. Cambridge Philos. Soc. 76, 61-66 (1974) 